NOTICE: this is the author's version of a work that was accepted for publication in Computers and Geosciences. Changes resulting from the publishing process, such as peer review, editing, corrections, structural formatting, and other quality control mechanisms may not be reflected in this document. Changes may have been made to this work since it was submitted for publication. A definitive version was subsequently published in Computers and Geosciences, [VOL31, ISSUE7, (2005)] http://dx.doi.org/10.1016/j.cageo.2005.01.014 


\title{
Which wavelet best reproduces the Fourier power
}

\author{
spectrum?
}

J F Kirby

\author{
Western Australian Centre for Geodesy \\ Curtin University of Technology \\ GPO Box U1987 \\ Perth WA 6845
}

Australia

tel: +61892667701

fax: +61 892662703

J.Kirby@curtin.edu.au

\begin{abstract}
The article compares the radially-averaged Fourier power spectrum against the global wavelet power spectrum ('global scalogram') for seven continuous, two-dimensional wavelets: Derivative of Gaussian, Halo, Morlet, Paul, Perrier and Poisson wavelets,
\end{abstract}


and a new wavelet based on a superposition of rotated Morlet wavelets, named the 'Fan' wavelet. This wavelet is complex, yet is able to give quasi-isotropic wavelet phase spectra. All seven wavelets were applied to synthetic and real data to test their ability to reproduce the Fourier spectrum: the Fan, Halo and Morlet wavelets reproduced this spectrum exactly; the Poisson wavelet performed very poorly. However, in tests of the space domain resolution of these wavelets with real and synthetic data, the Poisson wavelet gave by far the best results.

Key Words: Continuous wavelet transform, Fourier transform, isotropic wavelets. 


\section{Introduction}

The wavelet transform has increased in usage and popularity in recent years since its inception in the early 1980's, yet still does not enjoy the widespread usage of the Fourier transform. This situation is regrettable, as the wavelet transform has many advantages over its older cousin. As there are many good books and articles introducing the wavelet transform, this article will not delve into the theory behind wavelets. Recommended literature for the wavelet novice includes Addison (2002), Antoine et al. (2004), Chui (1992), Farge (1992), Foufoula-Georgiou and Kumar (1994), and Torrence and Compo (1998).

This article is concerned with the analysis of real-valued, two-dimensional (2D), spatially distributed data, $g(\mathbf{x})$, where $\mathbf{x}=(x, y)$ and $x$ could be longitude or grid eastings, and $y$ could be latitude or grid northings. However, any real-valued input data that are a function of two independent variables may be used. The convention employed in this article is that the domain in which the input data exists is referred to as $\mathbf{X}$-space, while its Fourier domain is referred to as $\mathbf{k}$-space, where $\mathbf{k}=(u, v)$ is radial wavenumber (spatial frequency).

The article also introduces a new, hybrid wavelet, the 'Fan wavelet' (also briefly described in Kirby and Swain, 2005), devised to yield quasi-isotropic wavelet phase information. Whereas real-valued wavelets are isotropic, they can only yield realvalued wavelet transforms, and hence cannot give phase information. Complex wavelets, however, while giving complex wavelet transforms (and hence wavelet phase), are anisotropic, only giving phase information in a specified direction. Hence, 
the Fan wavelet was developed to give virtually-isotropic wavelet phase information from a signal.

\section{The continuous wavelet transform: a summary}

Simply put, the wavelet transform enables a "power spectrum" to be calculated at each and every location of a specified signal, i.e., power as a function of space and frequency. Contrast this with the Fourier-derived power spectrum, which yields power as a function of frequency only - all spatial information has been hidden, or even lost in the case of radially-averaged power spectra. This advantage over the Fourier transform is achieved through the use of localised basis functions, rather than infinitely-repeating sines and cosines. The wavelet transform of a signal, $g(\mathbf{x})$, is computed from the convolution of the signal with the complex conjugate of a wavelet, $\psi(\mathbf{x})$. Hence, the wavelet transform is most often computed via the Fourier transform, making use of the convolution theorem of Fourier transforms (e.g., Bracewell, 1986), giving:

$$
\tilde{g}(s, \mathbf{x}, \theta)=\mathbf{F}^{-1}\left\{\hat{g}(\mathbf{k}) \hat{\psi}_{s, \theta}^{*}(\mathbf{k})\right\}
$$

Here, the $\tilde{g}(s, \mathbf{x}, \theta)$ are the resulting 'wavelet coefficients', $s$ is the dilation parameter ('scale'), $\mathbf{x}$ is the translation parameter, and $\theta$ is the rotation parameter. Also, $\mathbf{F}^{-1}$ is the inverse 2D Fourier transform, and $\hat{g}(\mathbf{k})$ is the Fourier transform of the signal. The $\hat{\psi}_{s, \theta}(\mathbf{k})$ is the Fourier transform of the 'daughter' wavelets: these are weighted, dilated, translated and rotated versions of a 'mother' wavelet, $\hat{\psi}(\mathbf{k})$, where:

$$
\hat{\psi}_{s, \theta}(\mathbf{k})=s \hat{\psi}\left(s \Omega^{-1}(\theta) \mathbf{k}\right)
$$


The rotation matrix, $\Omega(\theta)$, belongs to the $\mathrm{SO}(n)$ group (Farge, 1992), where $\theta$ determines the resolving azimuth of the wavelet. The scale determines the width (dilation) of the wavelet in $\mathbf{x}$-space, and hence determines resolution: at large scales, the coefficients reveal long wavelengths in the data; at small scales they show short wavelengths. In this regard, the wavelet transform resembles a series of band-pass filters. Hence, the translation of the wavelet over the signal, via the convolution integral, enables a region-by-region analysis of the signal. It is the combination of this translation with the wavelet dilation which allows the frequency characteristics at each location of the signal to be revealed.

However, the so-called 'Heisenberg uncertainty relation' of signal processing places limits on the wavelet transform. This relation is mathematically represented by:

$$
\Delta \mathrm{x} \Delta \mathrm{k} \geq 2 \pi
$$

where $\Delta \mathrm{x}$ is the precision or uncertainty of the wavelet coefficients in the space domain, and $\Delta \mathbf{k}$ is the precision in the wavenumber domain. This leads to an inverse proportionality between spatial and wavenumber precision. At large scales, wavelets tend to have a good wavenumber precision, but a poor spatial precision; conversely, at small scales they tend to have a poor wavenumber precision, but a good spatial precision.

It must be stressed that the presented article concerns the continuous wavelet transform (CWT), and not the discrete wavelet transform (DWT). Here, the 'continuous' or 'discrete' applies to the acceptable values of the dilation and translation parameters, not to the data (as it does in the Fourier case). The DWT demands 
that the dilations and translations assume only specified, discrete values, usually according to a power-of-two logarithmic scale, called a 'dyadic grid'. The dilation and translation parameters of the CWT, on the other hand, can assume any reasonable value on a continuum. Both the CWT and the DWT may be applied to discretelysampled data on a regular grid. Dyadic discrete wavelets yield no redundancy in the frequency content of the corresponding wavelet coefficients, hence the DWT is preferable when compressing or modelling data as it decomposes the signal into the smallest possible number of independent coefficients. Continuous wavelets, on the other hand, do have redundancy in the wavelet coefficients, but this can be put to good use in data analysis and interpretation.

It is also worth mentioning here the advantages the CWT has over the windowed Fourier transform (WFT), an example of which is the Gabor transform. Briefly, the Gabor transform uses a complex exponential that is modulated by a Gaussian envelope (window): while the frequency of the complex exponential can increase or decrease, the window width is fixed. A certain type of wavelet called the Morlet wavelet uses a similar basis function, but the difference is that the window width is variable (and directly proportional to wavelet scale), and the frequency of the complex exponential is linked to the width (where a large window implies low frequency, and vice versa). The corollary is that in contrast to the CWT, the WFT is not particularly good at analysing signals with a large bandwidth. [See, e.g., Addison (2002) for more information.] 


\section{Continuous wavelets}

Functions must fulfil two mathematical properties in order to be classified as 'wavelets'. These are: 1) they must have a zero mean value, called the 'admissibility condition', which can be expressed by:

$$
\iint_{-\infty}^{\infty} \psi(\mathbf{x}) d^{2} \mathbf{x}=0 \quad \text { or } \quad \hat{\psi}(0)=0
$$

and, 2) they must they must have finite energy:

$$
\iint_{-\infty}^{\infty}|\psi(\mathbf{x})|^{2} d^{2} \mathbf{x}=\iint_{-\infty}^{\infty}|\hat{\psi}(\mathbf{k})|^{2} d^{2} \mathbf{k}=0
$$

The real, continuous wavelets discussed here are even functions in both $\mathbf{x}$ - and k-spaces, so are isotropic, with no preferred direction which can be an advantage for certain data types or analysis purposes. However, real wavelets cannot reveal phase information as the wavelet coefficients have no imaginary component. Complex wavelets, in contrast, have an even real component, and an odd imaginary component, which means their Fourier transform is asymmetric (Bracewell, 1986). This property gives a directional dependence to complex wavelets, which can be used to reveal trends in data. It also means that complex wavelets are able to reveal phase information within a signal, whereas real wavelets cannot. The Fan wavelet, however, is a quasi-isotropic, complex wavelet constructed by averaging a series of complex wavelets over different directions.

The continuous mother wavelets implemented in the software are described below. As it is not always possible to write analytic expressions for $\psi(\mathbf{x})$, sometimes

only their Fourier transforms, $\hat{\psi}(\mathbf{k})$, are given, as it is in this form that they are computed. Cross-sectional profiles through these wavelets are shown in Fig. 1. 
Morlet: The 2D Morlet wavelet (Dallard and Spedding, 1993) is a complex wavelet, with associated directional dependence. In $\mathbf{x}$-space, its form is:

$$
\psi(\mathbf{x})=e^{i \mathbf{k}_{0} \cdot \mathbf{x}} e^{-|\mathbf{x}|^{2} / 2}
$$

shown in Fig. 2: it can be seen that there is a directional dependence to complex wavelets, which can be used to reveal trends in data. It is customary to choose $\mathbf{k}_{0}=\left(u_{0}, v_{0}\right)$ according to the desired analysis direction, while maintaining $\left|\mathbf{k}_{0}\right|=$ $\pi \sqrt{2 / \ln 2} \approx 5.336$, which ensures the admissibility condition is (almost) met. The Fourier transform of the Morlet wavelet is:

$$
\hat{\psi}(\mathbf{k})=e^{-\left|\mathbf{k}-\mathbf{k}_{0}\right|^{2} / 2}=e^{-\left[\left(u-\left|\mathbf{k}_{0}\right| \cos \theta\right)^{2}+\left(v-\left|\mathbf{k}_{0}\right| \sin \theta\right)^{2}\right] / 2}
$$

shown in Fig. 3, where $\theta$ is the (positive anticlockwise) angle made with the positive $u$-axis. Being complex, this wavelet is able to yield phase information, but only in the direction dictated by $\left(u_{0}, v_{0}\right)$.

Halo: This wavelet was devised by Dallard and Spedding (1993) as a real-valued 2D analogue of the Morlet wavelet. It has the Fourier transform:

$$
\hat{\psi}(\mathbf{k})=e^{-\left(|\mathbf{k}|-\left|\mathbf{k}_{0}\right|\right)^{2} / 2}
$$

with the value of $\left|\mathbf{k}_{0}\right|$ as for the Morlet wavelet. The Fourier transform of this wavelet is shown in Fig. 4. The $\mathbf{x}$-space form of the wavelet is shown in Fig. 5: note the absence of an imaginary part, and the isotropy of the wavelet. 
Derivative of Gaussian (DoG): The mother wavelet is the $m^{\text {th }}$ derivative of a Gaussian:

$$
\psi(\mathbf{x})=-\frac{d^{m}}{d|\mathbf{x}|^{m}}\left(e^{-|\mathbf{x}|^{2} / 2}\right)
$$

with Fourier transform:

$$
\hat{\psi}(\mathbf{k})=-(i|\mathbf{k}|)^{m} e^{-|\mathbf{k}|^{2} / 2}
$$

The DoG wavelets are real-valued if $m$ is even, and purely imaginary if $m$ is odd. The latter have limited applicability. For order $m=2$ (i.e., the second derivative of a Gaussian function), this wavelet is known as the 'Mexican hat' wavelet, or 'Marr' wavelet.

Paul: This real-valued wavelet is described in Perrier et al. (1995), with Fourier transform:

$$
\hat{\psi}(\mathbf{k})=e^{-m(\ln |\mathbf{k}|)^{2} / 2}=|\mathbf{k}|^{-m \ln |\mathbf{k}| / 2}
$$

the last term being faster to compute. The order, $m \geq 1$.

Perrier: Perrier et al. (1995) devised this real-valued wavelet specifically to analyse signals with exponential and power-law decreasing spectra. Its Fourier transform is:

$$
\hat{\psi}(\mathbf{k})=e^{-\left(|\mathbf{k}|^{2}+|\mathbf{k}|^{-2 m}\right) / 2}
$$

The order, $m \geq 1$. 
Poisson: This real-valued wavelet is described by Holschneider (2000). The mother wavelet is:

$$
\psi(\mathbf{x})=\frac{1-|\mathbf{x}|^{2}}{\left(1+|\mathbf{x}|^{2}\right)^{2}}
$$

with Fourier transform:

$$
\hat{\psi}(\mathbf{k})=|\mathbf{k}| e^{-|\mathbf{k}|}
$$

It has a close relationship with the Laplacian operator (ibid.), hence finding applications in potential field analysis. It should not be confused with the $1 \mathrm{D}$ complex Cauchy wavelets (ibid.), which are called 'Paul wavelets' by Farge (1992).

As can be seen from Fig. 1, the wavelets resemble bandpass filters, with the width of the 'bell' determining the wavenumber range resolved by the wavelet at a particular scale. It can be seen that the smaller the scale, the larger the wavenumber pass-band, in keeping with the uncertainty relation discussed in Section 2. This inherent property of the CWT means that small-scale wavelets actually resolve a sizeable proportion of larger-scale information. Fig. 1 shows that this phenomenon is particularly apparent for the DoG and Poisson wavelets, whereas the Halo and Fan/Morlet wavelets have the smallest amount of overlap.

\section{The Fan Wavelet}

\subsection{Superposing Morlet wavelets}

The idea of superposing Morlet wavelets was originally suggested by Farge (1992), and developed by Antoine et al. (1993), and Antoine and Murenzi (1996). The 
superposition is constructed by averaging a series of 2D Morlet wavelets [Eq. (7)] over a finite number of directions, $N_{\theta}$ :

$$
\hat{\psi}^{S}(\mathbf{k})=\frac{1}{N_{\theta}} \sum_{j=0}^{N_{\theta}-1} e^{-\left[\left(u-\left|\mathbf{k}_{0}\right| \cos \theta_{j}\right)^{2}+\left(v-\left|\mathbf{k}_{0}\right| \sin \theta_{j}\right)^{2}\right] / 2}
$$

where $\theta_{j}=j \delta \theta$, and $\delta \theta$ is the azimuthal increment between successive Morlet wavelets. This summation corresponds to moving the Morlet wavelet 'bump' along a circular locus in $\mathbf{k}$-space, for a given scale, and results in wavelet coefficients that are thus independent of $\theta$. The choice of $N_{\theta}$ and $\delta \theta$ determine whether we have isolated bumps, as in Antoine and Murenzi (1996), or a Fan wavelet, described later.

Consider the mechanics of taking the wavelet transform of real-valued data, $g(\mathbf{x})$, using a superposition of Morlet wavelets. Firstly, the Fourier transform of an asymmetric distribution of real-valued data is a 'Hermitian' function, with an even real part, $\hat{g}_{R}$, and an odd imaginary part, $\hat{g}_{I}$ (Bracewell, 1986). The three images in Fig. 6 show schematic diagrams of the imaginary part of this Fourier transform. If the upper-right quadrant in $\mathbf{k}$-space contains some distribution of numbers, $\mathbf{A}$, and the upper-left quadrant contains $\mathbf{B}$, then the lower quadrants are just negative reflections of these, - $\mathbf{A}$ and $\mathbf{- B}$, and hence contain redundant information.

Superimposed upon these quadrants in Fig. 6a is an annulus, representing multiplication by a Halo wavelet, $\hat{\psi}^{H}$. This can be expressed by Eq. (1), rewritten as:

$$
\mathbf{F}\left\{\tilde{g}^{H}(s, \mathbf{x})\right\}=\hat{g}_{R}(\mathbf{k}) \hat{\psi}_{s}^{H}(\mathbf{k})+i \hat{g}_{I}(\mathbf{k}) \hat{\psi}_{s}^{H}(\mathbf{k})
$$

since $\hat{\psi}^{H}$ has no imaginary part. As $\hat{g}_{R}$ and $\hat{\psi}^{H}$ are both even functions, and $\hat{g}_{I}$ is an odd function, $\mathbf{F}\{\tilde{g}\}$ is Hermitian, implying $\tilde{g}^{H}$ is real-only. This illustrates why 
real-valued, isotropic wavelets such as the Halo cannot yield the wavelet phase of a signal.

Fig. 6b shows multiplication of $\hat{g}_{I}$ by a superposition $\left(\hat{\psi}^{S}\right)$ of six Morlet wavelets $\left(\hat{\psi}^{M}\right)$, with $N_{\theta}=6, \delta \theta=60^{\circ}$, all equally spaced, and similar to the four wavelets in Antoine and Murenzi (1996):

$$
\begin{aligned}
\mathbf{F}\left\{\tilde{g}^{S}(s, \mathbf{x})\right\} & =\hat{g}_{R}(\mathbf{k}) \hat{\psi}_{s}^{S}(\mathbf{k})+i \hat{g}_{I}(\mathbf{k}) \hat{\psi}_{s}^{S}(\mathbf{k}) \\
& =\frac{1}{N_{\theta}} \sum_{j=0}^{N_{\theta}-1} \hat{g}_{R}(\mathbf{k}) \hat{\psi}_{s, \theta j}^{M}(\mathbf{k})+i \frac{1}{N_{\theta}} \sum_{j=0}^{N_{\theta}-1} \hat{g}_{I}(\mathbf{k}) \hat{\psi}_{s, \theta j}^{M}(\mathbf{k})
\end{aligned}
$$

using Eq. (15), the wavelet coefficients now being independent of $\theta$ owing to the averaging process. However, due to the anti-symmetry of $\hat{g}_{I}$, and since the bumps are diametrically opposed, the term $\sum \hat{g}_{I} \hat{\psi}^{M}$ sums to zero. Hence, from Eq. (17), $\mathbf{F}\left\{\tilde{g}^{S}\right\}$ is real-only, and therefore so are the wavelet coefficients, $\tilde{g}^{S}$. Thus no phase information is revealed, and this phenomenon occurs for all even values of $N_{\theta}$. For odd $N_{\theta}$ however, as the bumps are not diametrically opposed there is no cancellation of the imaginary part, and phase information can result. However, as $N_{\theta} \rightarrow \infty\left(N_{\theta}\right.$ odd), cancellation of the $\sum \hat{g}_{I} \hat{\psi}^{M}$ term does occur, since $N_{\theta}$ is so large as to cause $\hat{\psi}^{S}$ to span the whole space. In this limit, the large number of closely-located Morlet wavelets behave as the Halo wavelet.

\subsection{The Fan wavelet}

While the Fan wavelet is also a superposition of Morlet wavelets, as in Eq. (15), the azimuthal increment, $\delta \theta$, must be strictly controlled so as not to have discernable 
bumps:

$$
\delta \theta=\frac{2 \sqrt{-2 \ln p}}{\left|\mathbf{k}_{0}\right|}
$$

where $p$ represents the amplitude at which adjacent bell-curves intersect $(0<p<1)$. Fig. 7 shows a schematic diagram of the superposition of seven Morlet wavelets, for $p=0.75$. It was determined that this value of $p$ is optimal: any lower, and 'wiggles' appear in the superposition; any higher and too many Morlet wavelets are required to yield a desired azimuthal extent, $\Delta \theta$. The number of individual Morlet wavelets required to give a specific azimuthal extent is given by:

$$
N_{\theta}=\operatorname{int}\left(\frac{\Delta \theta}{\delta \theta}\right)
$$

Any value of $\Delta \theta$ may be used, though Section 4.3 illustrates the effect of varying this parameter between $0^{\circ}$ and $360^{\circ}$.

A perspective view of the Fourier transform of the Fan wavelet, for $\Delta \theta=180^{\circ}$, is shown in Fig. 8. It is worth mentioning that this geometry is very similar to that of the Arc wavelet [Dallard and Spedding, 1993, their Figure 4(c)], except that the Arc has an abrupt truncation at the $u$-axis. It is this discontinuity in the Arc that gives rise to the Gibbs phenomenon in the $\mathbf{x}$-space wavelet (ibid., their Figure 6), and renders the Arc not a wavelet in the strict sense. The real and imaginary parts of the Fan wavelet in $\mathbf{x}$-space are shown in Figs 9a and b respectively. Note the difference between the Halo wavelet (Fig. 5) and the real part of the Fan wavelet, the former being completely isotropic, while the latter is quasi-isotropic.

Fig. 6c shows multiplication of the afore-mentioned imaginary part of some arbitrary data, $\hat{g}_{I}(\mathbf{k})$, by a Fan wavelet of azimuthal extent $\Delta \theta=180^{\circ}$. Despite 
appearances, the Fan wavelet is still (practically) isotropic as it spans the whole of the upper two quadrants - remember that the lower quadrants contain no extra information. And except for effects caused by a slight leakage into the lower quadrants (discussed in Section 4.3), there is no cancellation of the $\sum \hat{g}_{I} \hat{\psi}^{M}$ term in Eq. (17). This means that $\mathbf{F}\{\tilde{g}\}$ is complex, as will be the wavelet coefficients. Hence, the Fan wavelet represents an improvement upon other superpositions of Morlet wavelets as it is both complex-valued and quasi-isotropic, and therefore able to yield omni-directional phase information.

\subsection{The effect of azimuthal extent on phase}

Fig. 6c showed a slight leakage of the Fan wavelet into the lower quadrants of $\mathbf{k}$ space, and according to the discussion in Section 4.2, this causes some cancellation of the $\sum \hat{g}_{I} \hat{\psi}^{M}$ term in Eq. (17). Owing to the smooth roll-off of the Gaussian function, this leakage is unavoidable if full isotropy (i.e., a complete span of the upper two quadrants) is desired, so in this Section the degree of cancellation, and its effect on the wavelet phase spectrum of a signal, will be quantified. Note that this leakage is what Dallard and Spedding (1993) sought to eliminate with the abrupt truncation of their Arc wavelet.

The degree of cancellation of this term can be determined by monitoring the ratio of the energies of the imaginary and real parts of the resulting wavelet coefficients, through a "complex energy ratio":

$$
Q_{C}(s)=\frac{\sum_{\mathbf{x}}\left[\tilde{g}_{I}(s, \mathbf{x})\right]^{2}}{\sum_{\mathbf{x}}\left[\tilde{g}_{R}(s, \mathbf{x})\right]^{2}}
$$


If the RMS value of this ratio over all scales in the wavelet transform is determined (i.e., $\left.Q_{C}(s) \rightarrow Q_{C}\right)$, and $Q_{C}$ then computed for Fan wavelet transforms of the signal at values of azimuthal extent ranging from $0^{\circ}$ to $360^{\circ}$, then plots such as Fig. 10 can be produced.

Fig. 10 is the $Q_{C}-\Delta \theta$ plot for the test signal. It can be seen that as long as $\Delta \theta \leq$ $160^{\circ}, Q_{C} \approx 1$ : i.e., real and imaginary parts of the wavelet coefficients have similar energies, implying there are very few cancellations of the $\sum \hat{g}_{I} \hat{\psi}^{M}$ term in Eq. (17). However, as $\Delta \theta$ increases beyond $160^{\circ}$, these cancellations become more frequent, causing this imaginary component to decrease in energy. At $\Delta \theta=180^{\circ}, Q_{C} \approx 0.8$. Finally, at $\Delta \theta=360^{\circ}$ there is complete cancellation (as in the Halo wavelet), and the imaginary term disappears. This decrease in the imaginary component of $\mathbf{F}\{\tilde{g}\}$ causes the signal's imaginary wavelet coefficients, $\tilde{g}_{I}$, to diminish also.

It was found that this decrease in the energy of the imaginary part affects the wavelet phase in magnitude only, and not in the relative positions of features in the phase spectrum. Hence, full isotropy can be achieved at the expense of a slight diminishing of the magnitude of the wavelet phase.

\section{The global wavelet power spectrum}

The wavelet power spectrum of a signal is often called a 'scalogram', and is the modulus-squared of the wavelet coefficients. In general, it is computed from:

$$
P_{g}^{W}(s, \mathbf{x}, \theta)=|\tilde{g}(s, \mathbf{x}, \theta)|^{2}
$$


where there is no $\theta$-dependence for real wavelets. The Fan wavelet scalogram, however, is computed from the average of a series of Morlet wavelet scalograms in a Fan geometry:

$$
P_{g}^{W F}(s, \mathbf{x})=\frac{1}{N_{\theta}} \sum_{j=0}^{N_{\theta}-1}\left|\tilde{g}^{M}(s, \mathbf{x}, \theta)\right|^{2}
$$

according to the constraints of the Fan wavelet, which actually gives a truer reflection of isotropy than computing the wavelet coefficients with a Fan wavelet, and then using Eq. (21).

A "global scalogram" may be determined by averaging the modulus of the wavelet coefficients over the whole study area, for each scale:

$$
P_{g}^{W}(s, \theta)=\frac{1}{N_{x} N_{y}} \sum_{\mathbf{x}} P_{g}^{W}(s, \mathbf{x}, \theta)
$$

which is now a function of scale, and (if applicable) azimuth. If the wavelet scales are converted to an equivalent Fourier wavenumber (Section 6), the global scalogram may be compared directly with the radially-averaged Fourier power spectrum (periodogram), which is computed from:

$$
P_{g}^{F}(|\mathbf{k}|)=\frac{1}{N_{x} N_{y}} \frac{1}{N_{a}} \sum_{|\mathbf{k}|}|\hat{g}(\mathbf{k})|^{2}
$$

where the summation is performed over annuli in the wavenumber domain, and $N_{a}$ is the number of power estimates in each annulus.

When complex wavelets are chosen to analyse the data, the wavelet phase may be computed from, in general:

$$
\phi_{g}^{W}(s, \mathbf{x}, \theta)=\tan ^{-1}\left[\frac{\tilde{g}_{I}(s, \mathbf{x}, \theta)}{\tilde{g}_{R}(s, \mathbf{x}, \theta)}\right]
$$


including the Fan wavelet, though this has no $\theta$-dependence. For real wavelets acting on real data, $\tilde{g}_{I}=0(\forall s, \mathbf{x}, \theta)$, giving no wavelet phase information.

\section{Equivalent Fourier wavenumber}

A mother wavelet, and all her daughters, being a kind of bandpass filter, may be assigned an equivalent Fourier wavenumber, $k_{F}$, which gives an idea of the frequency content of the signal being analysed. The relationship between $k_{F}$ and scale is:

$$
k_{F}=\frac{\kappa}{s}
$$

where $\kappa$ is a factor dependent upon the choice of wavelet, and the upon method used to find $k_{F}$. As has been stressed, there is no hard-and-fast relationship between the scale of an analysing wavelet and the signal's frequency content it portrays. Two schemes to relate these quantities are described below.

\subsection{Peak wavenumber method}

The simplest approach is to take the wavenumber at which the daughter wavelet has a maximum value, $k_{p}$, as being representative of the passband $\left(k_{F}=k_{p}\right)$. It is computed from the location in $\mathbf{k}$-space where the gradient of the Fourier transform of the wavelet is zero [for $\mathbf{k} \neq(0,0)]$ :

$$
\left.\nabla_{\mathbf{k}} \hat{\psi}_{s, \theta}(\mathbf{k})\right|_{k_{p}}=0
$$

where $\nabla_{\mathbf{k}}=(\partial / \partial u, \partial / \partial v)$. The second column in Table 1 shows the peak wavenumber for the discussed wavelets, with the value of $\kappa=s k_{p}$. 


\subsection{Maximum power method}

Meyers et al. (1993) devised a scheme whereby the equivalent Fourier wavenumber is determined by finding the wavelet transform of a sine wave of known wavenumber: $\mathbf{k}_{\delta}=\left(k_{\delta}, k_{\delta}\right)$. That value of $\mathbf{k}_{\delta}$ where the scalogram is a maximum gives $k_{F}$. Hence, if $g(\mathbf{x})=e^{i \mathbf{k}_{\delta} \cdot \mathbf{x}}$, its Fourier transform is $\hat{g}(\mathbf{k})=2 \pi^{2} \delta\left(\mathbf{k}_{\delta}-\mathbf{k}\right)$ (e.g. Bracewell, 1986), and from Eqs (1) and (2) its CWT is, for a given wavelet $\psi$ :

$$
\tilde{g}(s, \mathbf{x}, \theta)=s \hat{\psi}_{s, \theta}^{*}\left(\mathbf{k}_{\delta}\right) e^{i \mathbf{k}_{\delta} \cdot \mathbf{x}}
$$

The maximum power criterion is such that

$$
\frac{\partial}{\partial s}\left[P_{g}^{W}(s, \mathbf{x}, \theta)\right]=0
$$

which implies

$$
\frac{\partial}{\partial s}\left|s \hat{\psi}_{s, \theta}^{*}\left(\mathbf{k}_{\delta}\right)\right|^{2}=0
$$

Solving Eq. (30) for $k_{\delta}$, for a given wavelet, gives the equivalent Fourier wavenumber $\left(k_{F}=k_{\delta}\right)$. The third column in Table 1 shows the maximum-power wavenumber for the discussed wavelets, with the value of $\kappa=s k_{\delta}$.

\section{The 2D-CWT of Synthetic and Real Data}

In order to discern the applicability of the discussed wavelets, tests of their $\mathbf{x}$ and $\mathbf{k}$-space resolution were undertaken. For the $\mathbf{k}$-space analysis, the CWT of synthetic and real data was taken, and the global scalograms [Eq. (23)] compared with the corresponding radially-averaged Fourier power spectra [Eq. (24)]. The 
synthetic data were actually determined directly in k-space, while the real data are topography over Australia. Perrier et al. (1995) have conducted a similar study on synthetic data, though only on the DoG, Paul and Perrier wavelets. In the $\mathbf{x}$-space analysis, the scalograms of a synthetic test signal and the Australian topography were taken. The peak wavenumber method was used as an estimate of the equivalent Fourier wavenumber in all cases.

\subsection{Power-law spectrum}

Many physical and geophysical phenomena are fractal in nature (Turcotte, 1997), such as topography and the gravity field. These data have spectra with a power-law decay, such as:

$$
\hat{g}(\mathbf{k})=|\mathbf{k}|^{-\alpha}
$$

A value of $\alpha=3$ was used, implying a fractal dimension of 2.5. The global scalograms for the discussed wavelets are shown in Fig. 11 (dots), together with the function given by Eq. (31) (solid lines). It can be seen that the Fan and Halo wavelets perform best in mimicking the Fourier spectrum, closely followed by the Morlet and Perrier wavelets. The Paul wavelet overestimates the actual spectrum in the midwavelengths, the $2^{\text {nd }}$ order DoG wavelet more so. The Poisson wavelet does not reproduce the spectrum well at any wavelength. 


\subsection{Exponential spectrum}

Some geophysical data have spectra with an exponential decay, for example, the theoretical isostatic admittance function describing the state of Airy isostasy over a continent (e.g., Watts, 2001). The function used to test each wavelet in this case has the Fourier transform:

$$
\hat{g}(\mathbf{k})=e^{-|\mathbf{k}|}
$$

The global scalograms for the discussed wavelets are shown in Fig. 12 (dots), together with the function given by Eq. (32) (solid lines). As for the power-law spectrum, the Fan, Halo and Morlet wavelets best reproduce the Fourier spectrum. However, for this case the DoG, Perrier and Paul wavelets all underestimate the actual spectrum at the long and mid-wavelengths. Again, the Poisson wavelet fails to reproduce the Fourier spectrum at all wavelengths, showing a dramatic departure from this curve.

\subsection{A test signal}

The previous two studies, on power-law and exponential spectra, tested the response of the various wavelets in $\mathbf{k}$-space by analysing their global scalograms. Their behaviour in $\mathbf{x}$-space may be assessed by analysing the scalograms of a test signal, shown in Fig. 13a. This signal is of the form $\sin (10 \pi x / L) \sin (10 \pi y / L)$ to the 'east' of centre, $\sin (20 \pi x / L) \sin (20 \pi y / L)$ to the 'west' of centre (for $L=256 \mathrm{~km})$, and with the following additions: white noise added to a square region in the 'north'; a constant (DC) shift applied to another square region towards the 'south'; and a 
spike in the 'north-west'.

Figs $13 \mathrm{~b}-\mathrm{h}$ each show a slice, at $8 \mathrm{~km}$ equivalent Fourier wavelength, of the scalograms generated from the DoG $(m=2)$, Fan $\left(\Delta \theta=180^{\circ}\right)$, Halo, Morlet $\left(45^{\circ}\right.$ azimuth), Paul $(m=4)$, Perrier $(m=4)$ and Poisson wavelets, respectively. All wavelets successfully resolve the spike to varying degrees, the region of noise, and the frequency change from 'west' to 'east'. Being isotropic, the real wavelets (DoG, Halo, Paul, Perrier and Poisson) all resolve the edges of the DC-shifted region. The Morlet wavelet, however (Fig. 13e), only resolves the four corners of the DC-shifted region, due to the fact that the $45^{\circ}$ azimuth of the Morlet is not 'seeing' the edges, which are aligned at $0^{\circ}$ and $90^{\circ}$. The Fan wavelet (Fig. 13c), being isotropic, resolves features with any orientation as do the real wavelets, though its similarity with the Morlet wavelet is revealed in its not resolving the background sinusoids of the signal (though these are resolved in the wavelet phase image, discussed below). Undoubtedly, the Poisson wavelet (Fig. 13h) is the most effective wavelet at picking out the features of the test signal, with clear and abrupt delineation of the feature boundaries. Given the large width of the Fourier transform of the Poisson wavelet in Fig. 1, and the uncertainty relation, Eq. (3), this good spatial precision is not surprising. All other wavelets smear these boundaries, especially the Perrier wavelet (Fig. 13g), though the DoG wavelet shows only minimal smearing (Fig. 13b). Fourier transform edge effects are visible in all images toward the left and right axes, except that of the Poisson wavelet, another advantage of this wavelet, and only slightly in the DoG wavelet image.

Fig. 14a shows the Fan wavelet $\left(\Delta \theta=180^{\circ}\right)$ phase of the test signal, at $8 \mathrm{~km}$ 
equivalent Fourier wavelength. Fig. 14b shows the Morlet wavelet (45a azimuth) phase at the same wavelength. Both wavelets pick out the phase changes due to the spike, the region of noise, and the frequency change from 'west' to 'east'. However, as for their scalograms, the Fan wavelet resolves all the edges of the DC-shifted region, while the Morlet wavelet only resolves the four corners. Notice how the background sinusoids are resolved in the Fan wavelet phase image, whereas they were not in its scalogram. Also note how the contours of constant phase in the Morlet phase image are all aligned at $45^{\circ}$, in keeping with the chosen resolving azimuth of the Morlet.

Hence, the Fan wavelet combines the best features of Morlet and Halo wavelets: it is isotropic like the Halo (which cannot yield phase information), yet can give phase information as does the Morlet (which is not isotropic).

Finally, Fig. 15 shows the Perrier wavelet scalogram of the test signal. This image has eastings and northings in $\mathrm{km}$ along the two horizontal axes ( $\mathrm{x}$ - and $\mathrm{y}$-axes, respectively), and equivalent Fourier wavelength in $\mathrm{km}$ (using the peak wavenumber method) on the vertical (z) axis, though the scale on this axis is logarithmic. Short wavelengths (small scales) are plotted towards the top of the z-axis, long wavelengths (large scales) towards the bottom. The displayed image shows slices taken in the $\mathrm{x}-\mathrm{Z}$ plane, and in the $\mathrm{x}-\mathrm{y}$ plane. The $\mathrm{x}-\mathrm{z}$ slice shows the relative power in the signal as a function of easting and equivalent Fourier wavelength (wavelet scale), at a northing of $200 \mathrm{~km}$. The frequency change from west to east, the spike, and region of noise are all clear. The $\mathrm{x}-\mathrm{y}$ slice shows the relative power in the signal of features having a wavelength of $32 \mathrm{~km}$. However, owing to the non-orthogonality of the CWT, it is not a perfect bandpass filter, and hence this slice will contain the signals of features 
in a range around this wavelength. Nevertheless, it can be seen that the smearing and ringing evident at $8 \mathrm{~km}$ wavelength (Fig. 13g) in the Perrier wavelet is not so manifest at $32 \mathrm{~km}$ wavelength.

\subsection{Australian topography}

Topographic elevation data over Australia were also used to test the algorithm, and to compare wavelets. The data are from the GEODATA 9-arcsecond DEM (digital elevation model) of Australia, produced by Geoscience Australia [1], regridded at $6^{\prime} \times 6^{\prime}$, and shown in Fig. 16a.

The $\mathbf{x}$-space resolution of the wavelets when applied to these data is determined by analysing their scalograms. Figs $16 \mathrm{~b}-\mathrm{h}$ each show a slice, at a scale of $21 \mathrm{~km}$, of the scalograms generated from the DoG $(m=2)$, Fan $\left(\Delta \theta=180^{\circ}\right)$, Halo, Morlet (45 azimuth), Paul $(m=4)$, Perrier $(m=4)$ and Poisson wavelets, respectively. Recalling that wavelets act as bandpass filters, the Poisson wavelet appears to yield the best image (Fig. 16h), though it is apparent that a broad range of wavelengths appear in the image. This, again, is due to the uncertainty relation giving a poor wavenumber resolution but a good spatial resolution. As for the test signal, the DoG wavelet shows a reasonably good resolution of the topographic features at this scale, while the Fan and Halo wavelets both perform adequately. The Perrier wavelet gives the worst image, with a great deal of edge effects around the continent.

The phase images from the complex wavelets are shown in Figs 17a and b, for the Fan wavelet $\left(\Delta \theta=180^{\circ}\right)$ and Morlet wavelet ( $45^{\circ}$ azimuth), respectively. While the Morlet wavelet phase is strongly aligned at $45^{\circ}$ azimuth, the Fan phase is isotropic, 
picking up phase changes of the topographic features in all directions.

Comparing the global scalograms of the DEM with the radially-averaged Fourier power spectra, Fig. 18 shows the former as dots, and the latter as solid lines, on log-log plots. As can be seen, the signal's spectrum obeys a power-law decrease characteristic of fractal data. All wavelets, except the Poisson wavelet, succeed in reproducing the Fourier spectrum, though slight departures are exhibited with the Paul and Perrier wavelets, and with the DoG wavelet at high wavenumbers. The Morlet global scalogram shows a large dip at low wavenumbers, presumably due to the DEM not having much power in this part of the spectrum at the chosen azimuth $\left(45^{\circ}\right)$. Interestingly, at low wavenumbers, the wavelets provide a better representation of the fractal nature of the topography than does the Fourier spectrum, with the lowest-wavenumber estimate of power in the latter departing from a straight line.

\section{Discussion}

The resolution of seven continuous wavelets was tested in the space $(\mathbf{x})$ and wavenumber (k) domains. In $\mathbf{k}$-space, this was achieved by comparing their global scalograms with radially-averaged Fourier power spectra for a synthetic power-law spectrum, a synthetic exponentially-decaying spectrum, and real Australian topographic data.

The Fan, Halo and Morlet wavelets were the most successful at reproducing the Fourier spectra. This is to be expected, however, as these wavelets are, in the space domain, complex exponentials modulated by a Gaussian envelope, hence being very 
similar to the Fourier basis functions.

The $2^{\text {nd }}$ order DoG, and $4^{\text {th }}$ order Paul and Perrier wavelets all yielded over- or under-estimates of the Fourier spectra. This is somewhat surprising for the Perrier wavelet, which was designed specifically to model such signals. In $\mathbf{x}$-space, the DoG showed only a small degree of smearing over feature boundaries, whereas the Perrier wavelet yielded very poor scalogram images at small scales, exhibiting a large amount of ringing, though this decreased at large scales.

The Poisson wavelet consistently failed to duplicate the Fourier spectra, a rather worrying fact since this wavelet has been used in a number of gravity-field applications. A glance at Fig. 1 explains this phenomenon: the Halo and Fan wavelets have much less overlap at adjacent scales than does the Poisson, which smears the frequency content at each scale to a much greater amount, particularly at the smaller scales. This property is not desirable in spectral analysis, and means the Poisson wavelet has a very limited applicability in this area. However, when a space domain analysis is desired, the Poisson is perhaps the most useful of the discussed wavelets, with clearly defined edges at phase changes within the signals. Again, this result is not surprising as the implications of the uncertainty relation mean its poor $\mathbf{k}$-space resolution gives a good $\mathbf{x}$-space resolution.

Concerning wavelet phase, real wavelets cannot generate such information, while the Morlet wavelet is restricted to phase in one specified azimuth. Hence, a complex wavelet has been constructed which yields practically-isotropic wavelet phase information for real-valued, two-dimensional data. This has been achieved by accounting for the redundancy of the two lower quadrants in the wavenumber domain, 
and superposing a number of rotated Morlet wavelets in the upper two quadrants only. The resulting Fan wavelet is not an even function, thus generating imaginary wavelet coefficients for a given, real-valued, signal.

For a desired azimuthal extent, or angular resolving power, of less than $160^{\circ}$ the fan wavelet preserves the relative magnitude of real and imaginary parts of the wavelet coefficients. If complete isotropy is desired in the wavelet transform, i.e. an azimuthal extent of $180^{\circ}$, then the energy in the imaginary part of the wavelet coefficients will be approximately $80 \%$ of the energy in their real part, though this does not affect the spatial location of features in the phase spectrum. This energy ratio decreases to zero at an azimuthal extent of $360^{\circ}$, in keeping with expectations for real wavelets.

The Fortran90 source code used to compute the wavelet transforms, and the Matlab scripts to generate Fig. 15, are described in the Appendix, and are available from the Computers and Geosciences website.

\section{Acknowledgements}

I would like to thank Tom Ridsdill-Smith for discussions while developing the CWT code, Chris Swain and Irek Baran for "sexing-up" my Matlab code, and Jo Ward for discussions. I am grateful to the National Mapping Division of Geoscience Australia for supplying the 9 arc-second DEM. 


\section{References}

Addison, P.S., 2002. The Illustrated Wavelet Transform Handbook. Institute of Physics Publishing, Bristol, UK, 353pp.

Antoine, J.-P., Carrette, P., Murenzi, R., Piette, B., 1993. Image analysis with two-dimensional continuous wavelet transform. Signal Processing 31, 241-272.

Antoine, J.-P., Murenzi, R., 1996. Two-dimensional directional wavelets and the scale-angle representation. Signal Processing 52, 259-281.

Antoine, J.-P., Murenzi, R., Vandergheynst, P., Ali, S.T., 2004. Two-dimensional Wavelets and their Relatives. Cambridge University Press, Cambridge, UK.

Bracewell, R.N., 1986. The Fourier transform and its applications, 2nd edn. McGrawHill, New York, USA, 474pp.

Chui, C.K., 1992. An Introduction to Wavelets. Academic Press, San Diego, USA, 266pp.

Dallard, T., Spedding, G.R., 1993. 2-D wavelet transforms: generalisation of the Hardy space and application to experimental studies. European Journal of Mechanics, B/Fluids 12(1), 107-134.

Farge, M., 1992. Wavelet transforms and their applications to turbulence. Annual Review of Fluid Mechanics 24, 395-457.

Foufoula-Georgiou, E., Kumar, P. (Eds), 1994. Wavelets in Geophysics. Academic Press, San Diego, USA, 372pp.

Holschneider, M., 2000. Introduction to continuous wavelet analysis. In: Klees, R., Haagmans, R. (Eds), Wavelets in the Geosciences. Springer-Verlag, Berlin, 1-71. 
Kirby, J.F., 2002. A FORTRAN90 subroutine to calculate array sizes prior to a mixed-radix fast Fourier transform. Computers and Geosciences 28, 999-1001.

Kirby, J.F., Swain, C.J., 2004. Global and local isostatic coherence from the wavelet transform. Geophysical Research Letters, 31(24), L24608, doi: 10.1029/2004GL021569. Meyers, S.D., Kelly, B.G., O'Brien, J.J., 1993. An introduction to wavelet analysis in oceanography and meteorology: with application to the dispersion of Yanai waves. Monthly Weather Review 121, 2858-2866.

Perrier, V., Philipovitch, T., Basdevant, C., 1995. Wavelet spectra compared to Fourier spectra. Journal of Mathematical Physics 36, 1506-1519.

Torrence, C., Compo, G.P., 1998. A practical guide to wavelet analysis. Bulletin of the American Meteorological Society 79(1), 61-78.

Turcotte, D.L., 1997. Fractals and Chaos in Geology and Geophysics, 2nd edn. Cambridge University Press, Cambridge, UK, 398pp.

Watts, A.B., 2001. Isostasy and Flexure of the Lithosphere. Cambridge University Press, Cambridge, UK, 458pp.

\section{Internet References}

[1] GEODATA 9 Second DEM Version 2 User Guide. Geoscience Australia. https://www.ga.gov.au/image_cache/GA4783.pdf 
Figure 1: Cross-sectional profiles through the Fourier transforms of the seven discussed wavelets: scale $s=60$ (solid line), $s=40$ (dashed), $s=20$ (dotted). Note the abscissa scale change for the Halo and Morlet/Fan wavelets.

Figure 2: The Morlet wavelet in $\mathbf{x}$-space, at a resolving azimuth of $45^{\circ}$ : (a) real, and (b) imaginary parts. Horizontal axes are $x$ and $y$ coordinates.

Figure 3: The Fourier transform of the Morlet wavelet, at a resolving azimuth of $45^{\circ}$. Horizontal axes are $u$ and $v$ wavenumbers. The Fourier transform of the Halo wavelet.

Figure 4: The Fourier transform of the Halo wavelet.

Figure 5: The Halo wavelet in $\mathbf{x}$-space.

Figure 6: The imaginary part of $\hat{g}$, multiplied by (a) a Halo wavelet, (b) a 6-fold Morlet superposition, (c) a Fan wavelet of $\Delta \theta=180^{\circ}$.

Figure 7: The superposition (solid line) of seven Morlet wavelets (dashed lines), for $p=0.75$.

Figure 8: The Fourier transform of the Fan wavelet, for $\Delta \theta=180^{\circ}$.

Figure 9: The Fan wavelet in $\mathbf{x}$-space: (a) real, and (b) imaginary parts. $\Delta \theta=180^{\circ}$.

Figure 10: A graph of the variation of the complex energy ratio, $Q_{C}$ (RMS over all scales), with azimuthal extent, $\Delta \theta$, of the Fan wavelet, for the test signal in Fig. $13 \mathrm{a}$. 
Figure 11: The global wavelet scalograms (dots) corresponding to the function $|\mathbf{k}|^{-3}$ (solid line), for seven wavelets.

Figure 12: The global wavelet scalograms (dots) corresponding to the function $e^{-|\mathbf{k}|}$ (solid line), for seven wavelets.

Figure 13: The test signal (a), and its scalograms at $8 \mathrm{~km}$ equivalent Fourier wavelength, for (b) DoG, (c) Fan, (d) Halo, (e) Morlet, (f) Paul, (g) Perrier, and (h) Poisson wavelets. Grey-scale for scalograms is $\log _{10}$ (power), from high (light) to low (dark) power.

Figure 14: The wavelet phase of the test signal (Fig. 13a) at $8 \mathrm{~km}$ equivalent Fourier wavelength, for (a) Fan, and (b) Morlet wavelets. Grey-scale is from $+90^{\circ}$ (light) to $-90^{\circ}$ (dark)

Figure 15: The Perrier wavelet scalogram of the test signal (Fig. 13a). The vertical axis is equivalent Fourier wavelength $(\mathrm{km})$. Grey-scale is $\log _{10}($ power $)$, from high (light) to low (dark) power.

Figure 16: The Australian DEM (a), and its scalograms at $21 \mathrm{~km}$ scale, for (b) DoG, (c) Fan, (d) Halo, (e) Morlet, (f) Paul, (g) Perrier, and (h) Poisson wavelets. Grey-scale for scalograms is $\log _{10}$ (power), from high (light) to low (dark) power.

Figure 17: The wavelet phase of the Australian DEM (Fig. 16a) at $80 \mathrm{~km}$ scale, for (a) Fan, and (b) Morlet wavelets. Grey-scale is from $+90^{\circ}$ (light) to $-90^{\circ}$ (dark).

Figure 18: Log-log plots of global wavelet scalograms (dots) and radially-averaged 
Fourier power spectrum (solid line) of the $6^{\prime}$ DEM of Australia. 

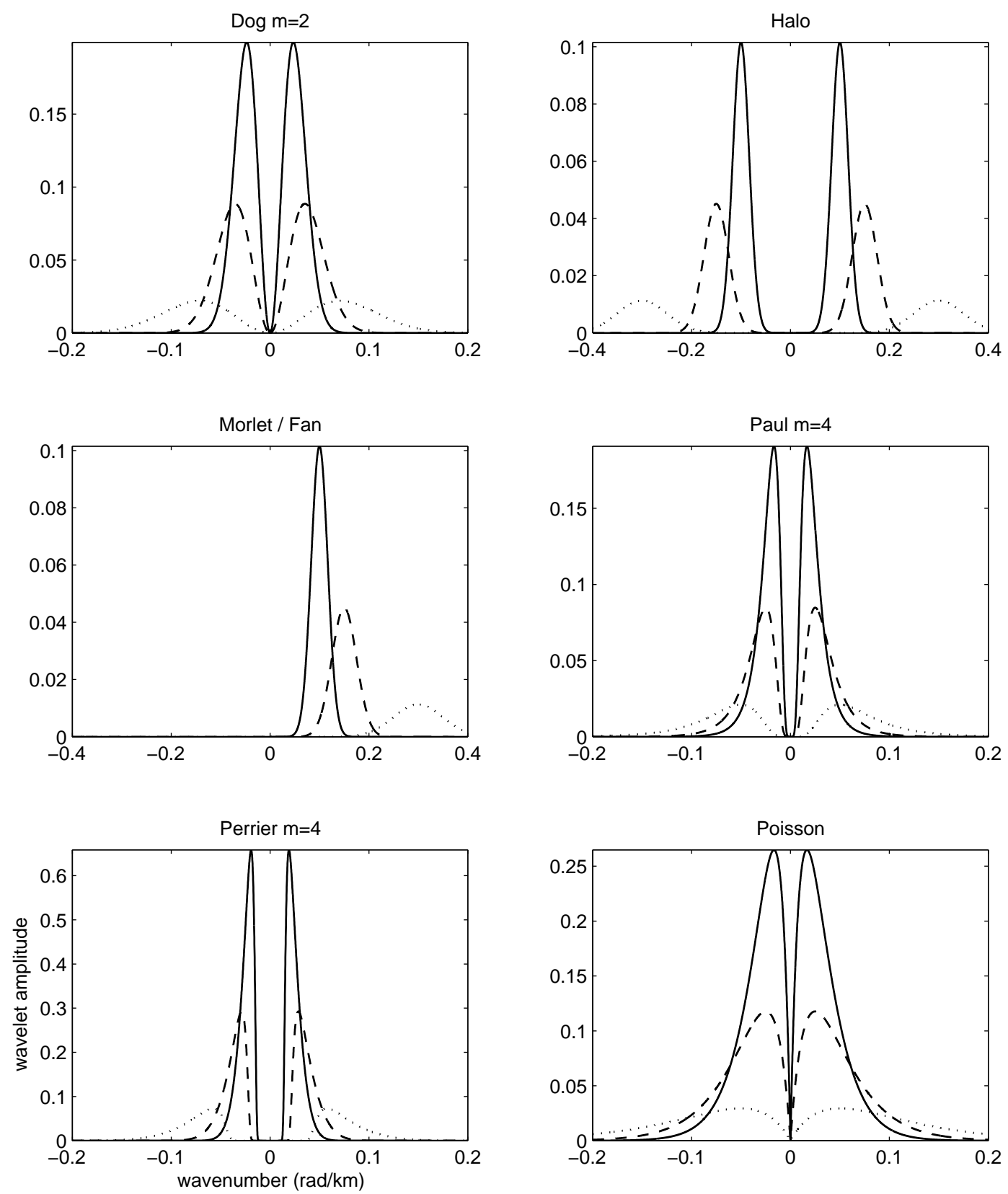

Figure 1: Cross-sectional profiles through the Fourier transforms of the seven discussed wavelets: scale $s=60$ (solid line), $s=40$ (dashed), $s=20$ (dotted). Note the abscissa scale change for the Halo and Morlet/Fan wavelets. 

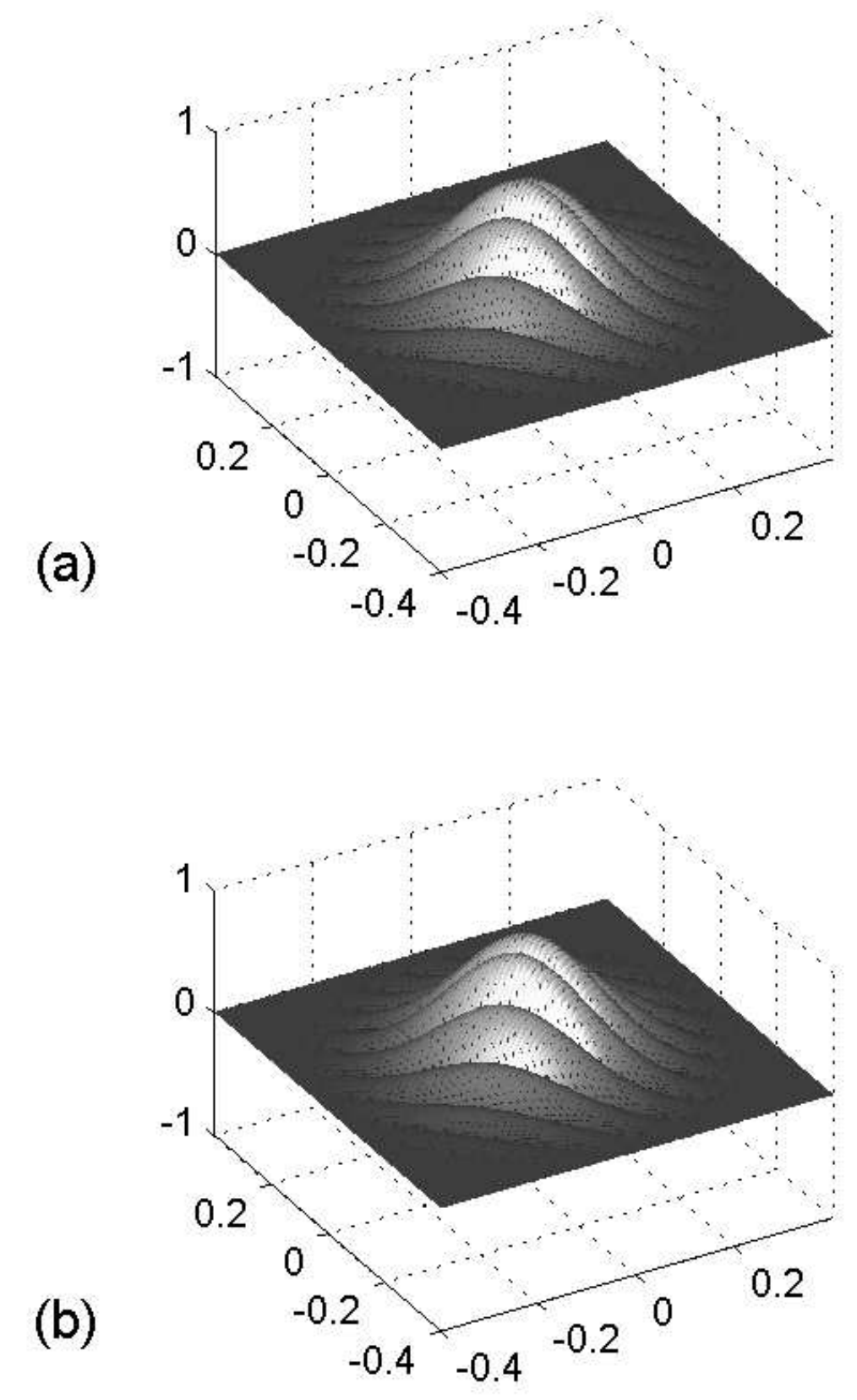

Figure 2: The Morlet wavelet in $\mathbf{x}$-space, at a resolving azimuth of $45^{\circ}$ : (a) real, and (b) imaginary parts. Horizontal axes are $x$ and $y$ coordinates. 


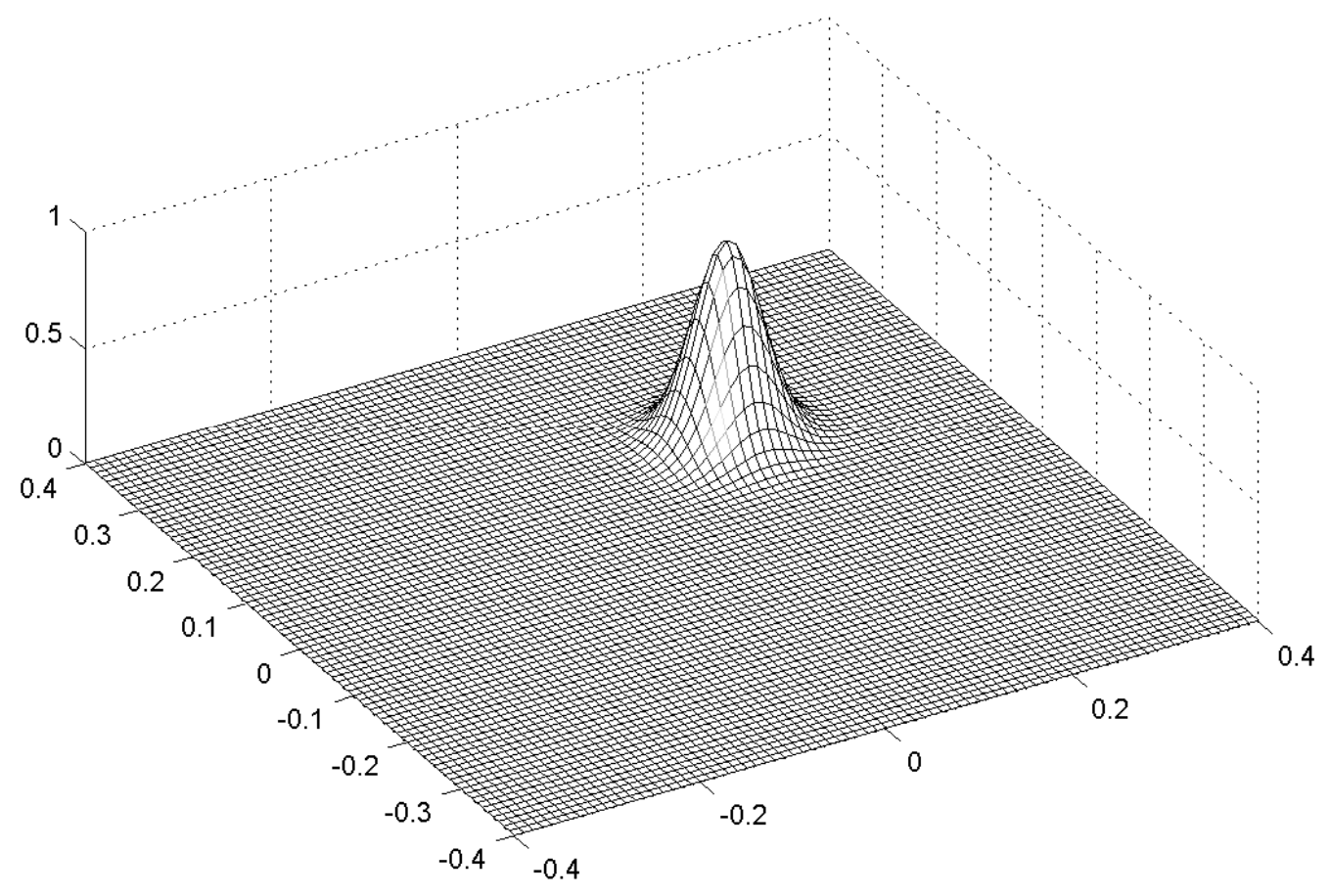

Figure 3: The Fourier transform of the Morlet wavelet, at a resolving azimuth of $45^{\circ}$. Horizontal axes are $u$ and $v$ wavenumbers. 


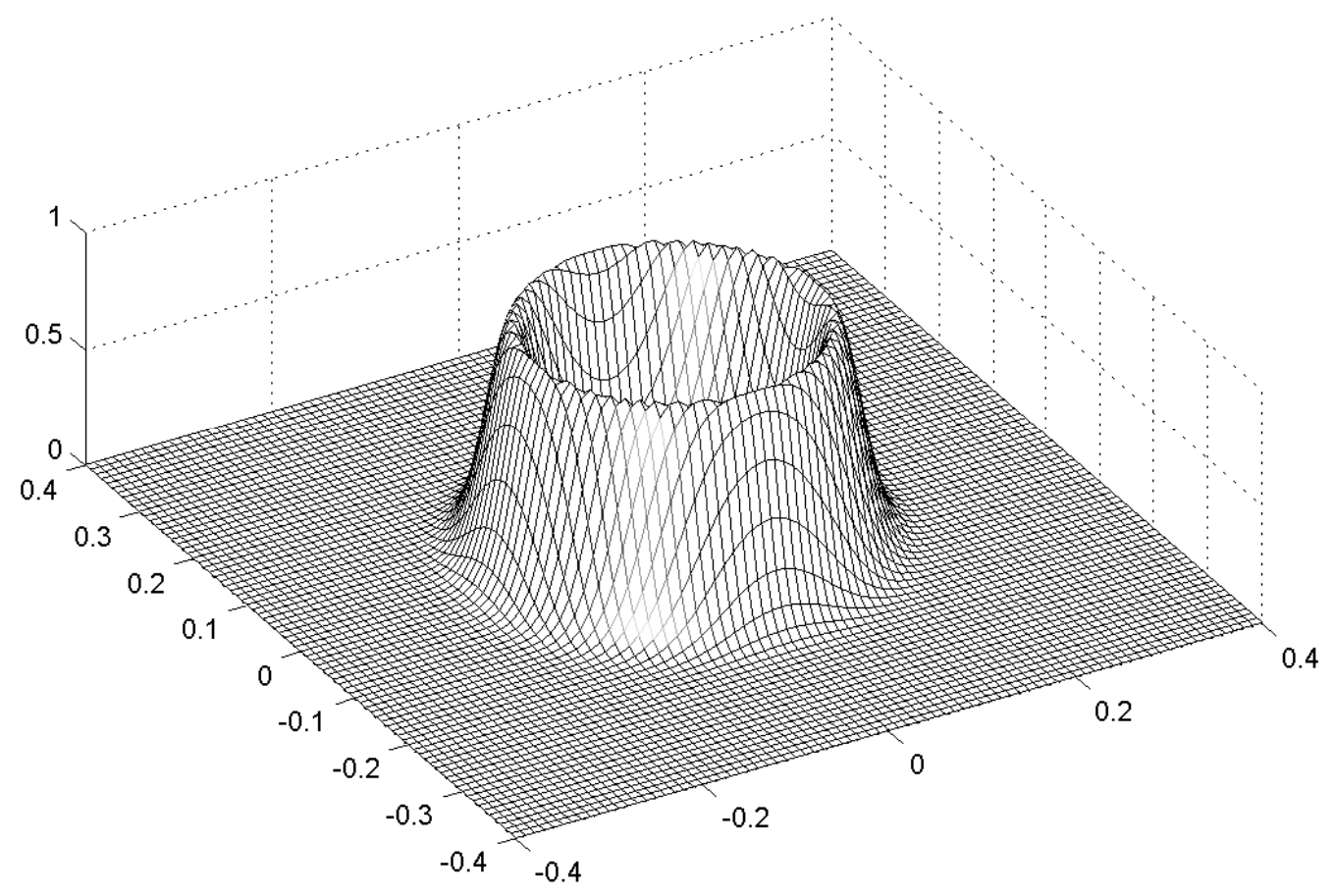

Figure 4: The Fourier transform of the Halo wavelet. 


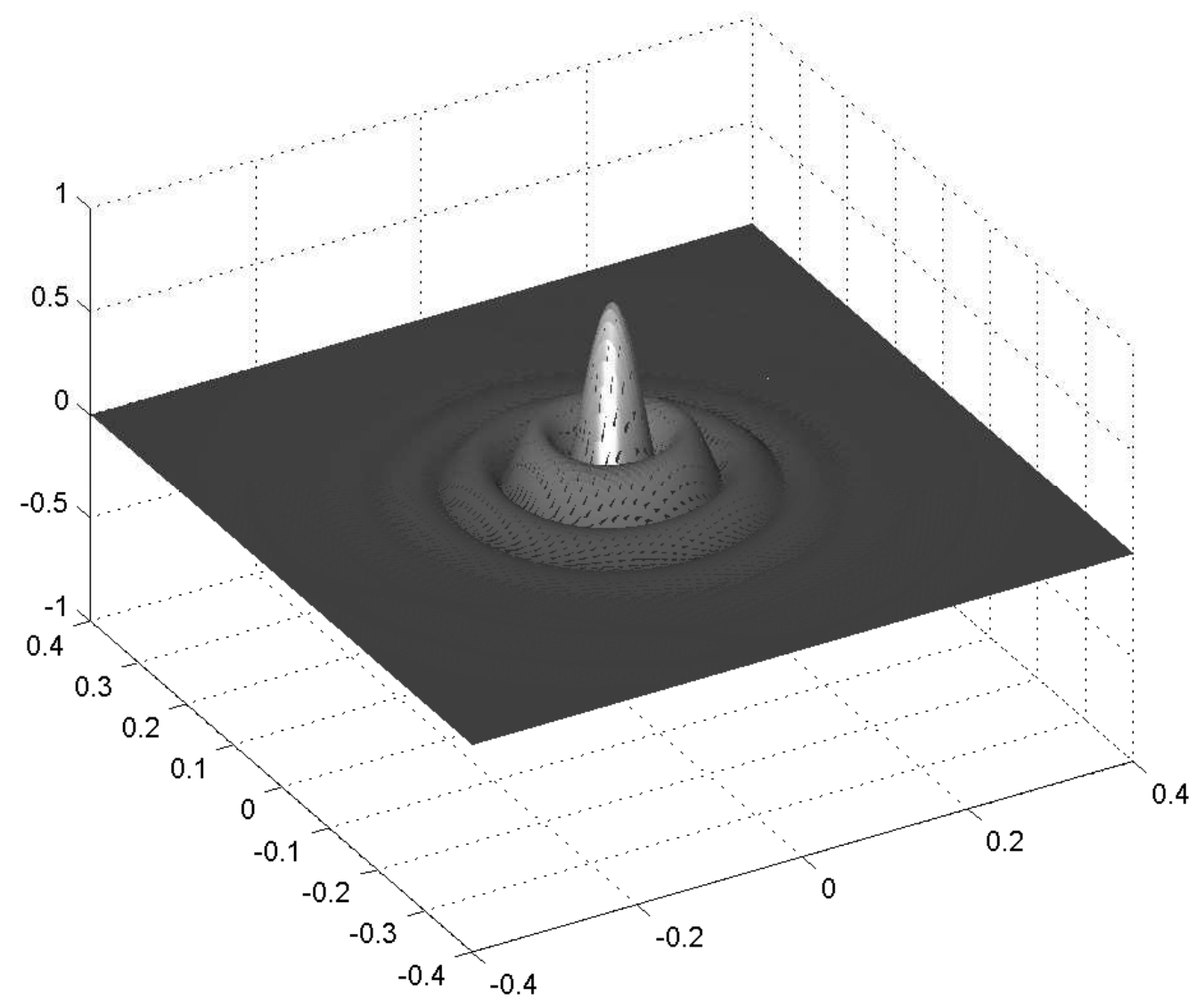

Figure 5: The Halo wavelet in $\mathbf{x}$-space. 
(a)

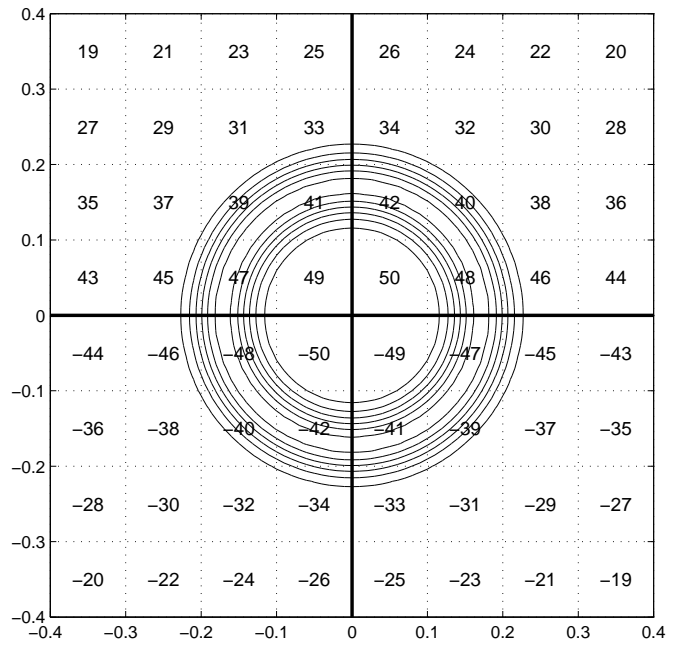

(b)

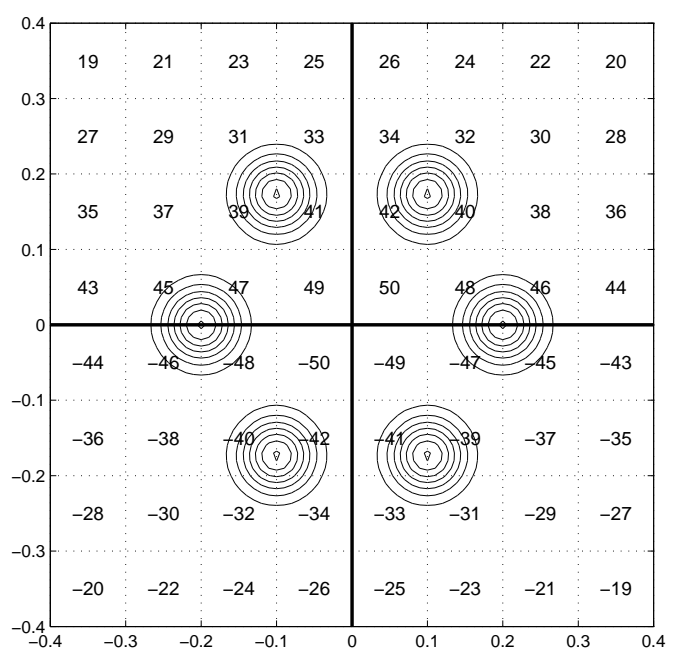

(c)

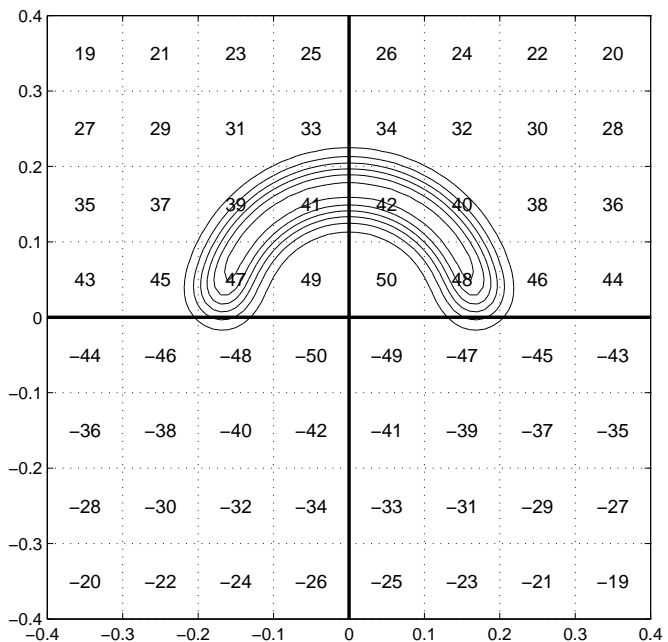

Figure 6: The imaginary part of $\hat{g}$, multiplied by (a) a Halo wavelet, (b) a 6 -fold Morlet superposition, (c) a Fan wavelet of $\Delta \theta=180^{\circ}$. 


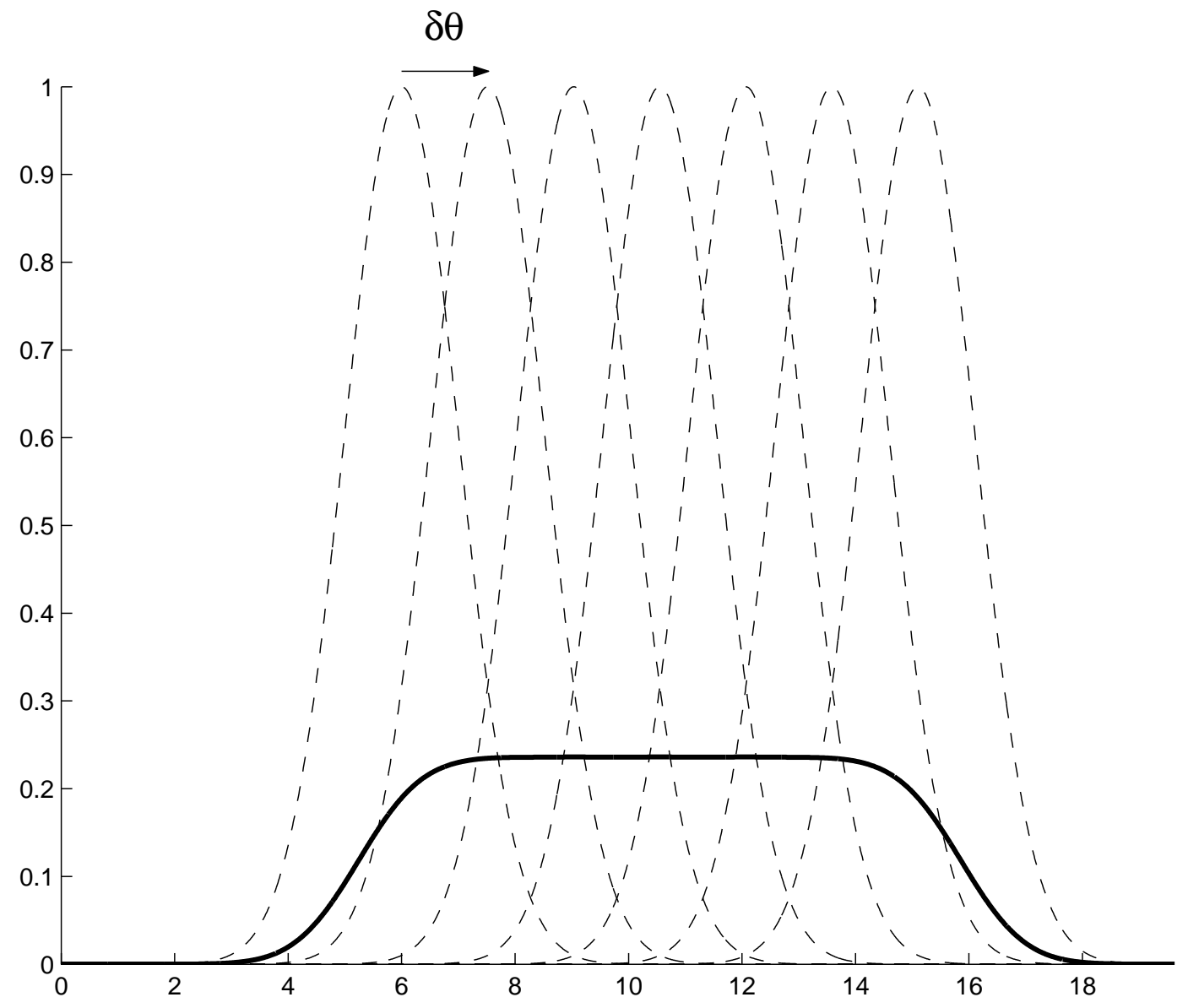

Figure 7: The superposition (solid line) of seven Morlet wavelets (dashed lines), for $p=0.75$. 


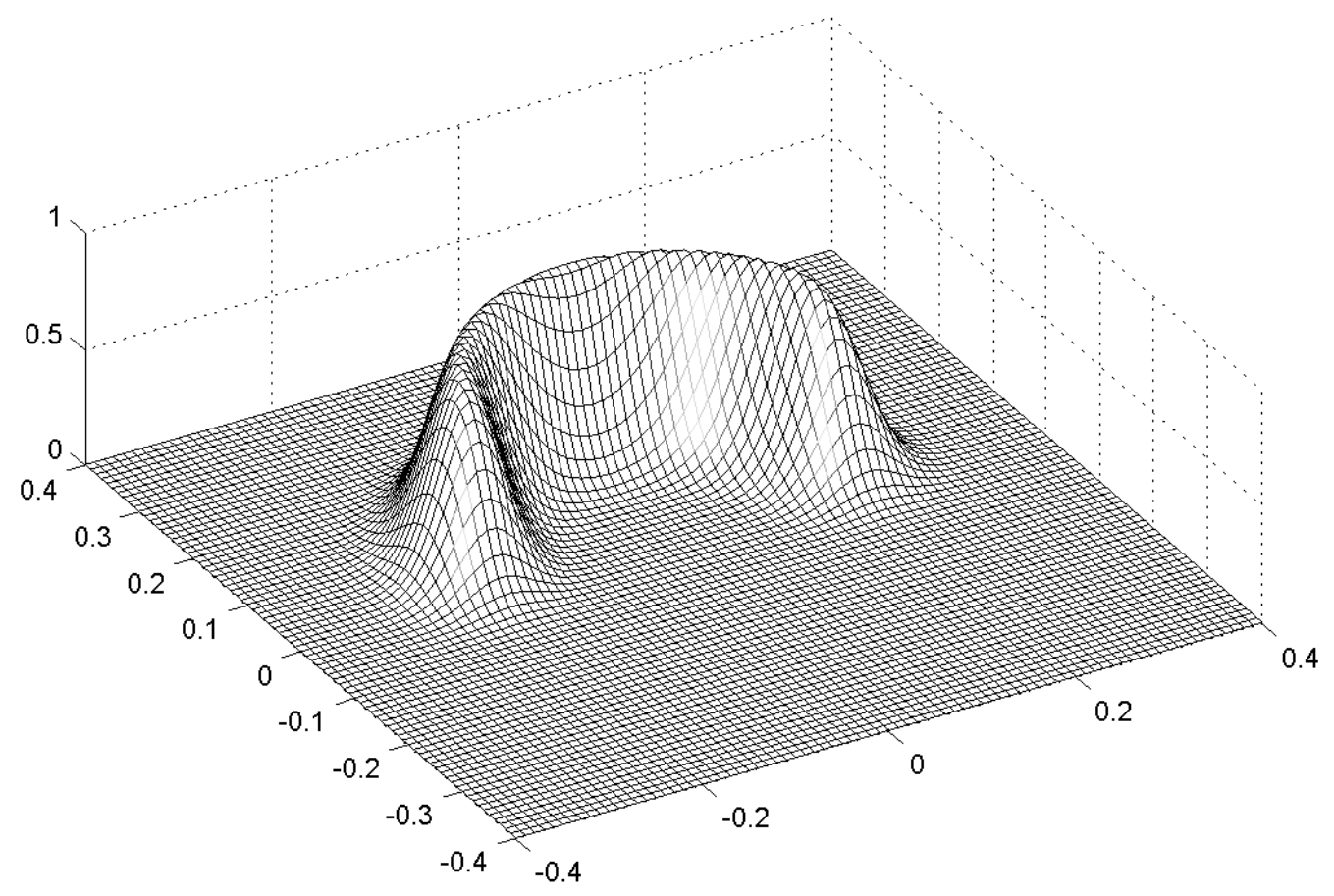

Figure 8: The Fourier transform of the Fan wavelet, for $\Delta \theta=180^{\circ}$. 

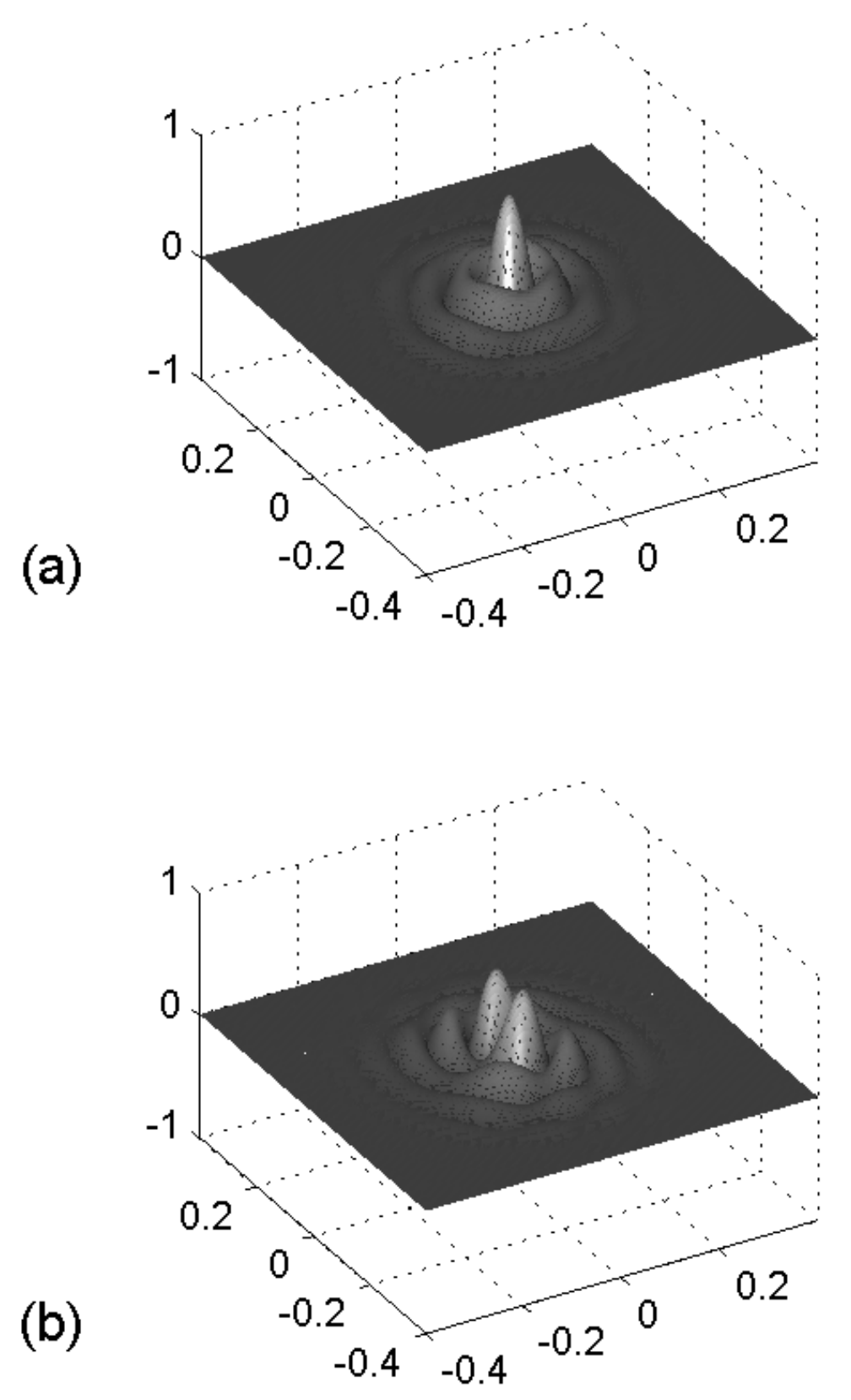

Figure 9: The Fan wavelet in $\mathrm{x}$-space: (a) real, and (b) imaginary parts. $\Delta \theta=180^{\circ}$. 


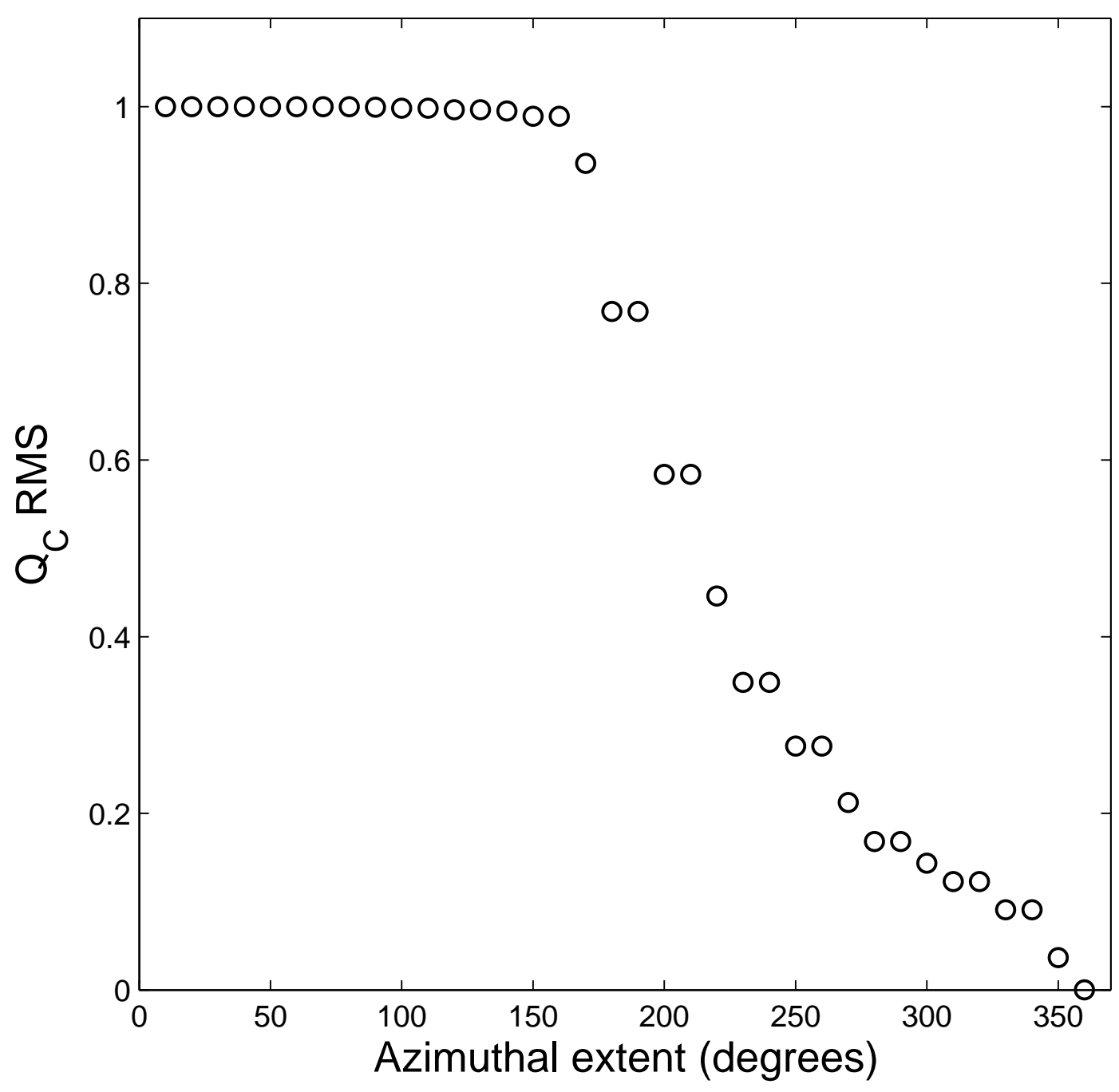

Figure 10: A graph of the variation of the complex energy ratio, $Q_{C}$ (RMS over all scales), with azimuthal extent, $\Delta \theta$, of the Fan wavelet, for the test signal in Fig. $13 \mathrm{a}$. 

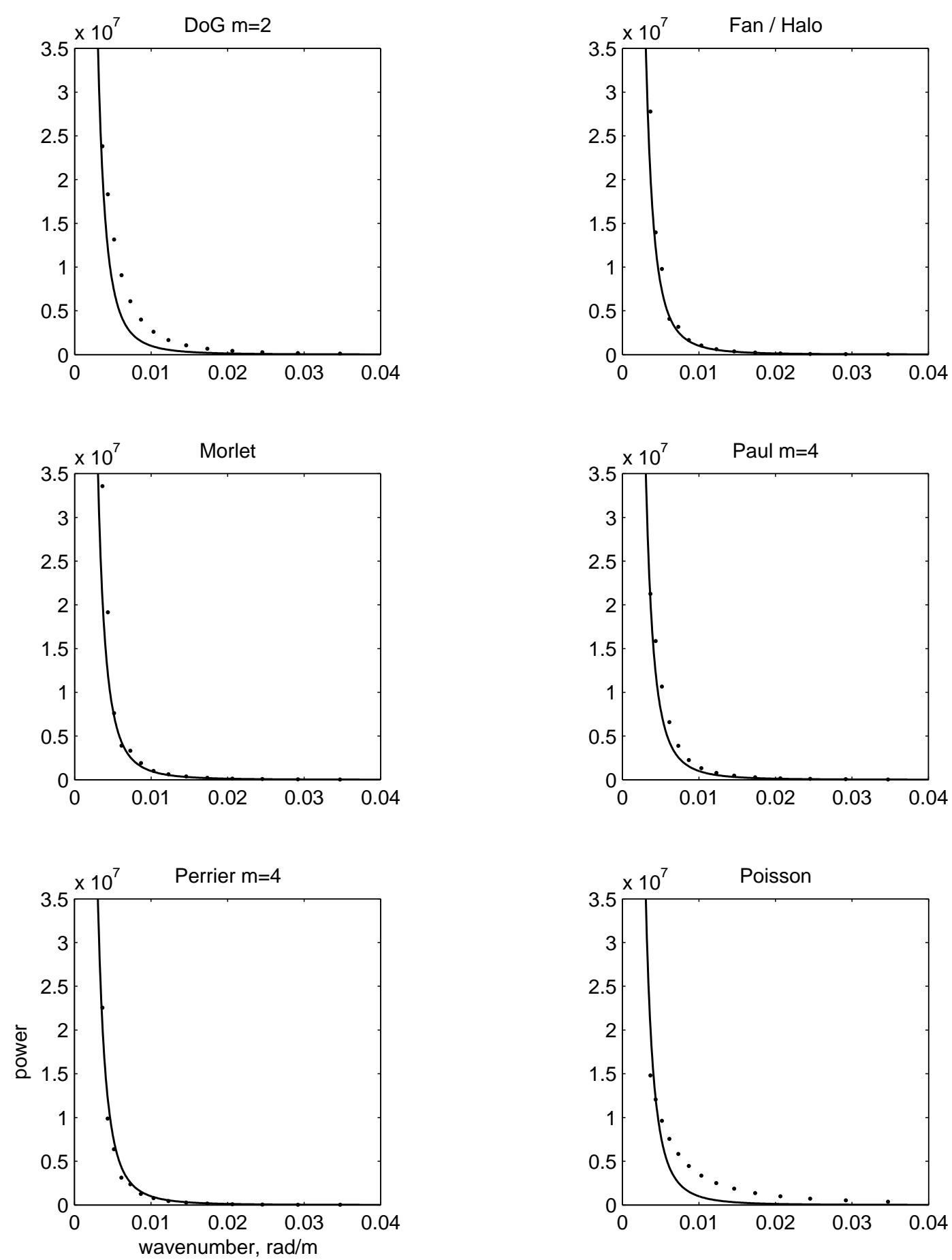

Figure 11: The global wavelet scalograms (dots) corresponding to the function $|\mathbf{k}|^{-3}$ (solid line), for seven wavelets. 

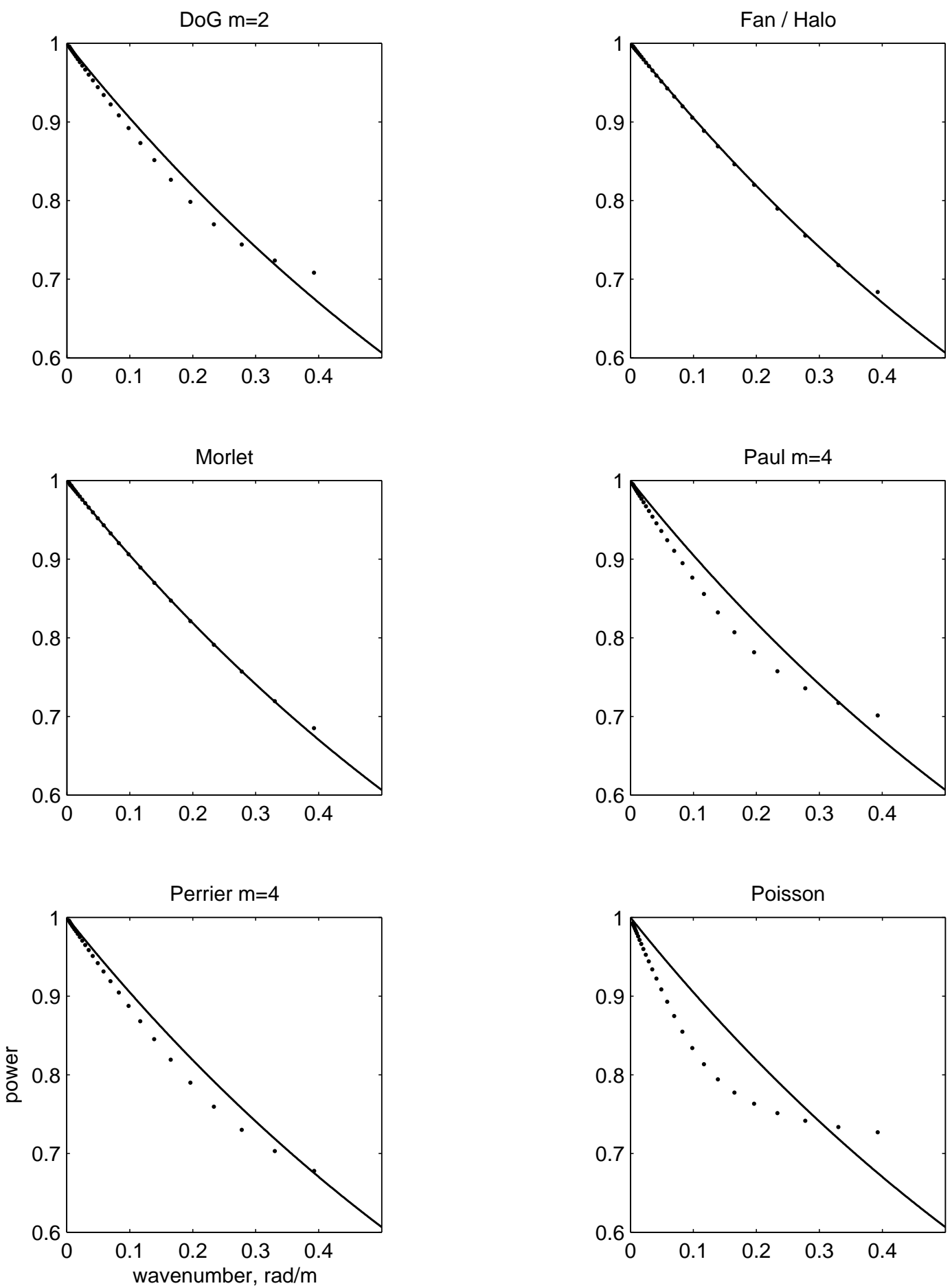

Figure 12: The global wavelet scalograms (dots) corresponding to the function $e^{-|\mathbf{k}|}$ (solid line), for seven wavelets. 

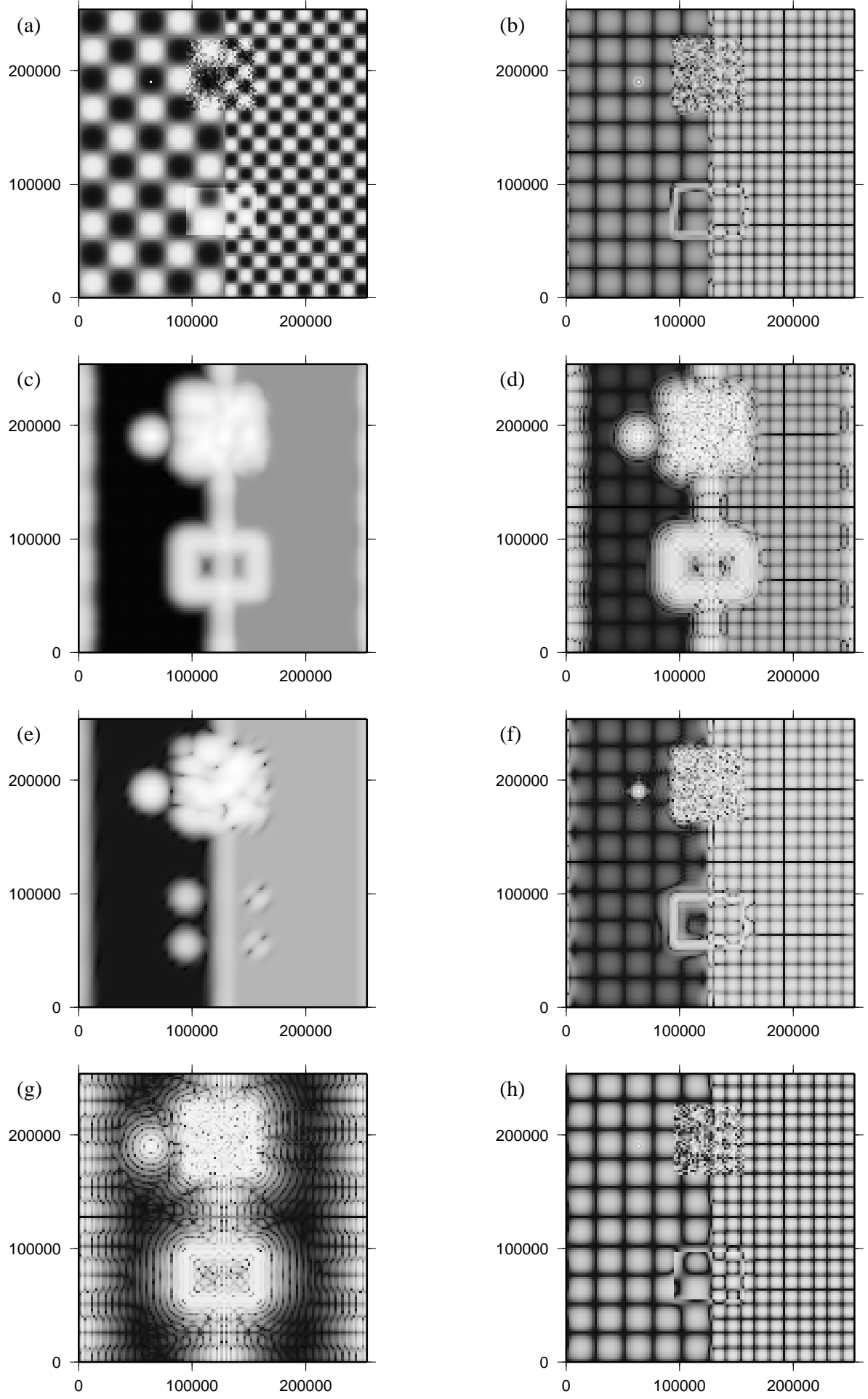

Figure 13: The test signal (a), and its scalograms at $8 \mathrm{~km}$ equivalent Fourier wavelength, for (b) DoG, (c) Fan, (d) Halo, (e) Morlet, (f) Paul, (g) Perrier, and (h) Poisson wavelets. Grey-scale for scalograms is $\log _{10}$ (power), from high (light) to low (dark) power. 

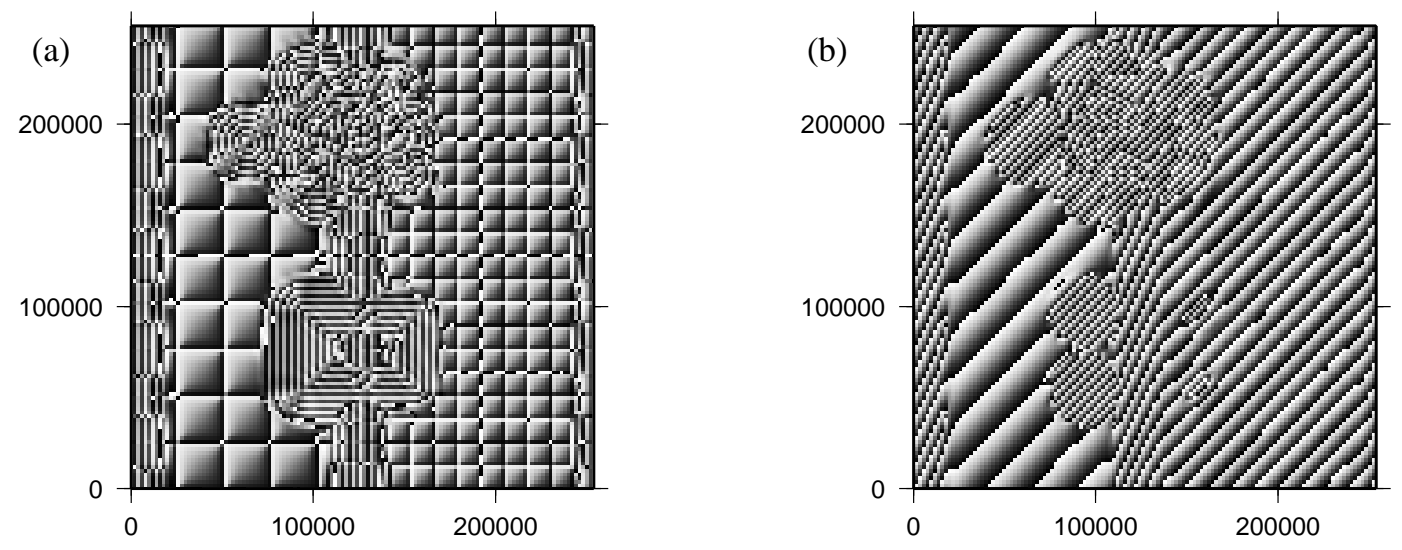

Figure 14: The wavelet phase of the test signal (Fig. 13a) at $8 \mathrm{~km}$ equivalent Fourier wavelength, for (a) Fan, and (b) Morlet wavelets. Grey-scale is from $+90^{\circ}$ (light) to $-90^{\circ}$ (dark) 


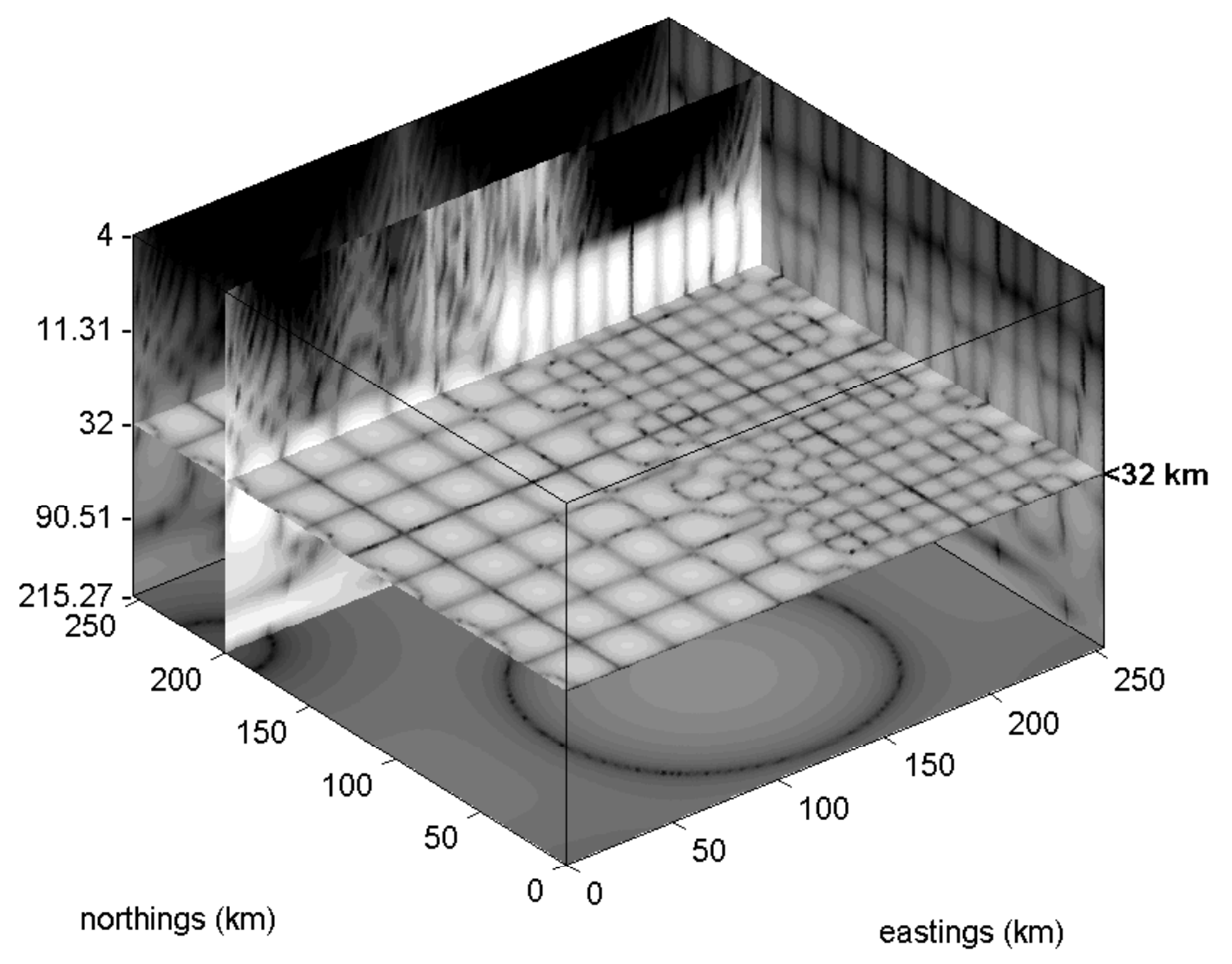

Figure 15: The Perrier wavelet scalogram of the test signal (Fig. 13a). The vertical axis is equivalent Fourier wavelength $(\mathrm{km})$. Grey-scale is $\log _{10}($ power$)$, from high (light) to low (dark) power. 
(a)

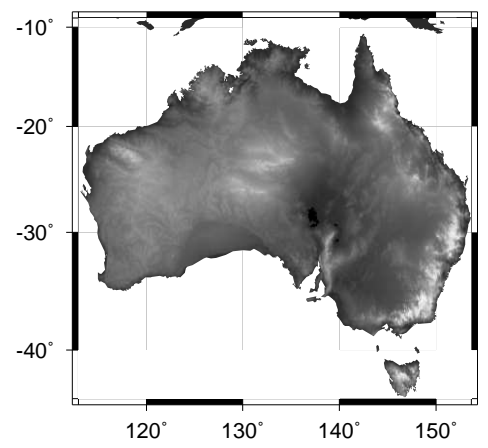

(c)

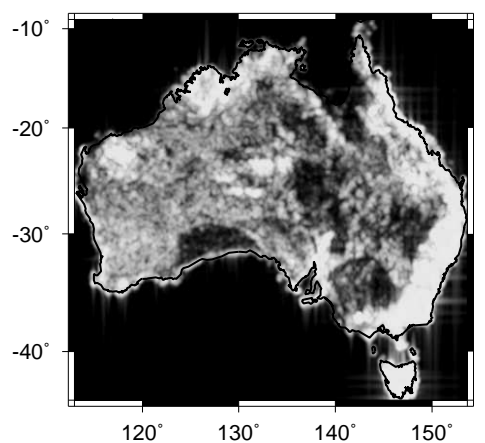

(e)

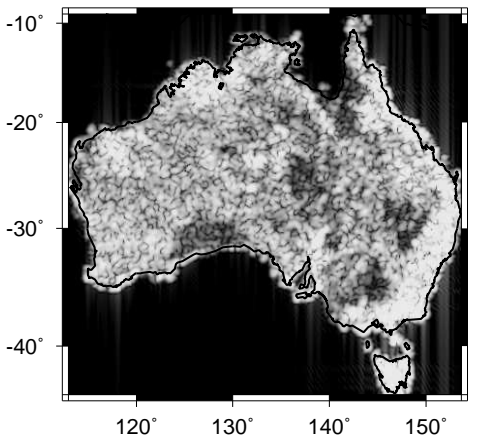

(g)

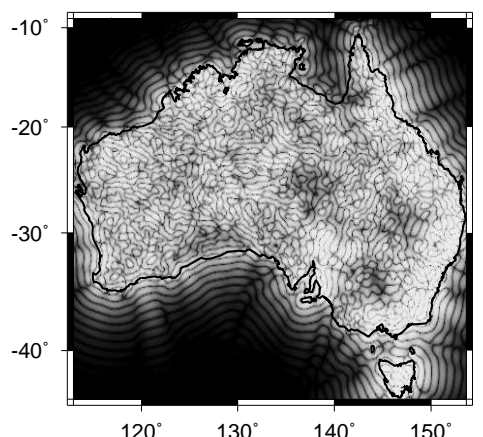

(b)

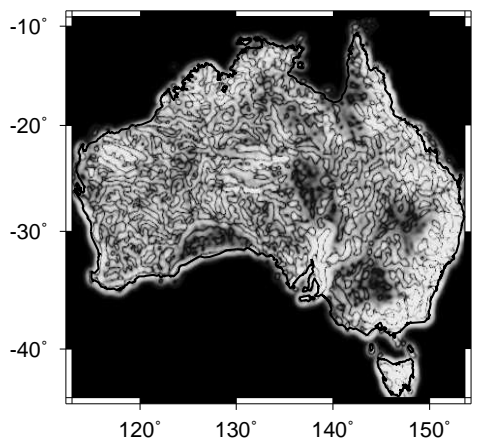

(d)

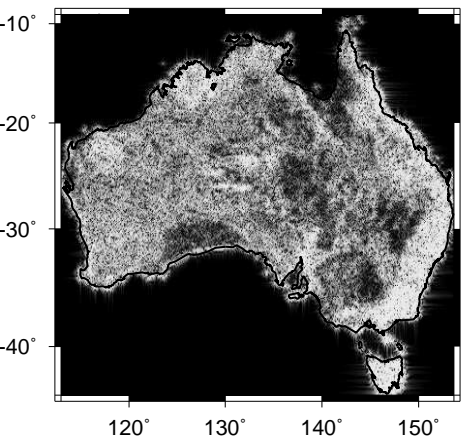

(f)

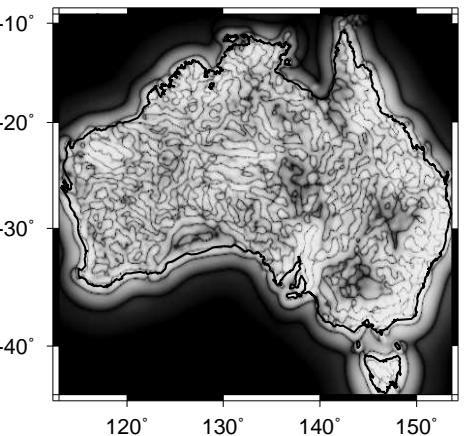

(h)

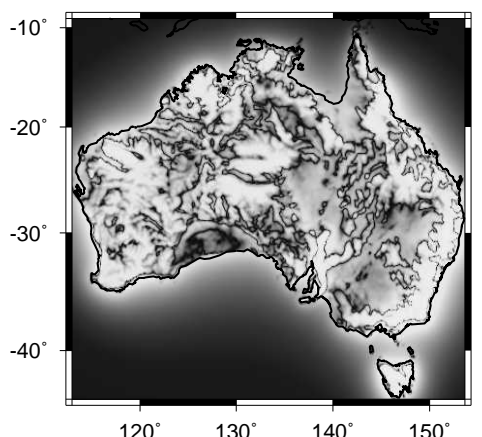

Figure 16: The Australian DEM (a), and its scalograms at $21 \mathrm{~km}$ scale, for (b) DoG, (c) Fan, (d) Halo, (e) Morlet, (f) Paul, (g) Perrier, and (h) Poisson wavelets. Grey-scale for scalograms is $\log _{10}$ (power), from high (light) to low (dark) power. 

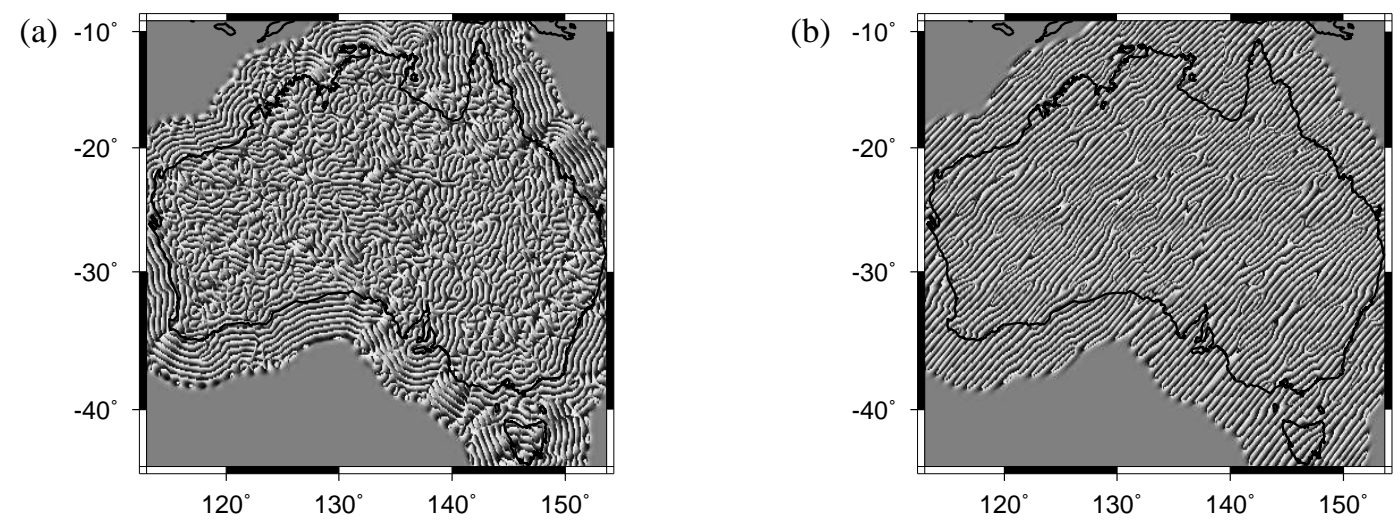

Figure 17: The wavelet phase of the Australian DEM (Fig. 16a) at $80 \mathrm{~km}$ scale, for (a) Fan, and (b) Morlet wavelets. Grey-scale is from $+90^{\circ}$ (light) to $-90^{\circ}$ (dark). 

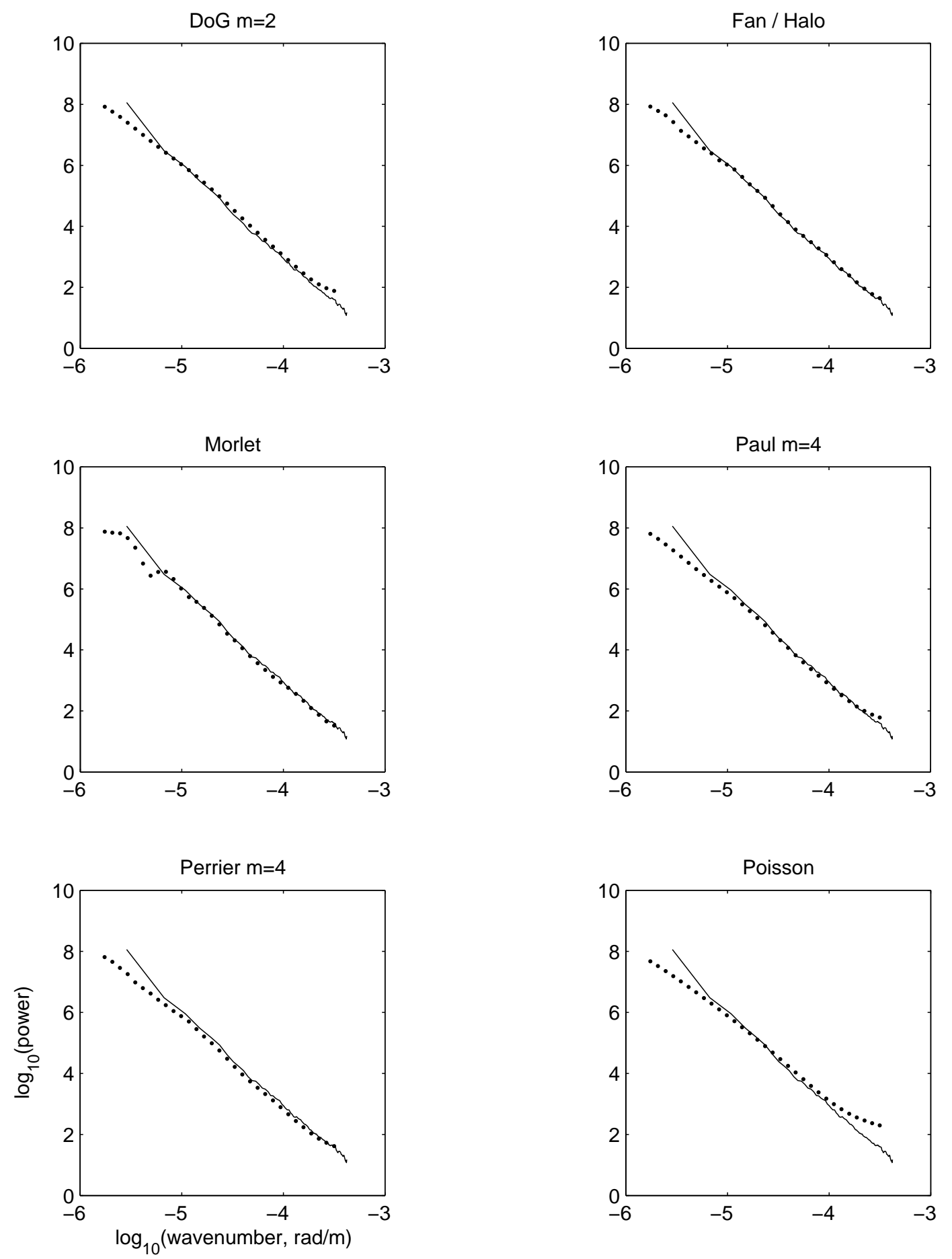

Figure 18: Log-log plots of global wavelet scalograms (dots) and radially-averaged Fourier power spectrum (solid line) of the $6^{\prime}$ DEM of Australia. 
Table 1: Equivalent Fourier wavenumbers, $k_{F}$. Choose $k_{F}=k_{p}$ for peak wavelet wavenumber representation, or $k_{F}=k_{\delta}$ for maximum power wavenumber representation. The value of $k_{\delta}$ for the Perrier wavelet is obtained from the solution to $2 s^{2} k_{\delta}^{2 m+2}-k_{\delta}^{2 m}-2^{-m} m s^{-2 m}=0$. The value of $\kappa=s k_{F}$. The equivalent Fourier wavelength may be found from $\lambda_{F}=2 \pi / k_{F}$. 


\begin{tabular}{ccc}
\hline & $k_{p}$ & $k_{\delta}$ \\
\hline DoG & $\frac{\sqrt{m}}{s}$ & $\frac{\sqrt{m+1}}{s \sqrt{2}}$ \\
Morlet/Fan & $\frac{k_{0}}{s}$ & $\frac{\left(u_{0}+v_{0}\right)+\sqrt{8+\left(u_{0}+v_{0}\right)^{2}}}{4 s}$ \\
Halo & $\frac{k_{0}}{s}$ & $\frac{k_{0}+\sqrt{4+k_{0}^{2}}}{2 s \sqrt{2}}$ \\
Paul & $\frac{1}{s}$ & $\frac{e^{1 / m}}{s \sqrt{2}}$ \\
Perrier & $\frac{m^{1 /(2 m+2)}}{s}$ & see below \\
Poisson & $\frac{1}{s}$ & $\frac{\sqrt{2}}{s}$ \\
\hline
\end{tabular}

Table 1: Equivalent Fourier wavenumbers, $k_{F}$. Choose $k_{F}=k_{p}$ for peak wavelet wavenumber representation, or $k_{F}=k_{\delta}$ for maximum power wavenumber representation. The value of $k_{\delta}$ for the Perrier wavelet is obtained from the solution to $2 s^{2} k_{\delta}^{2 m+2}-k_{\delta}^{2 m}-2^{-m} m s^{-2 m}=0$. The value of $\kappa=s k_{F}$. The equivalent Fourier wavelength may be found from $\lambda_{F}=2 \pi / k_{F}$. 


\section{Appendix - Software}

\section{cwt $2 d . f$}

This Fortran90 program computes the continuous wavelet transform of any complete and regularly gridded data set which is real-valued, and a function of two variables. It is primarily written for data that are geographically distributed, but any data that are functions of two independent variables are acceptable. The source code is well-commented, with opening lines that inform the user of all input requirements, and output information. At the end of the source code is a sample command file (which must be called cwt2d.com).

The file cwt $2 \mathrm{~d}$.f contains all subroutines called by the main program. These are primefac.f (Kirby, 2002), detrend2d.f, demean2d.f, mirror2d.f, taper2d.f, and fft.f. A mean value is always removed from the data, but it remains up to the user to determine whether it is necessary to 1) pad the data with zeros, 2) detrend the data (by removal of a best-fitting plane), 3) reflect the data at its edges, or 4) apply a $5 \%$ cosine taper to its edges, in order to reduce the adverse effects of Fourier transformation [see Bracewell (1986) for a full discussion]. The author recommends experimentation.

The program was written for a Sun Workshop 6 Fortran90 compiler. The execution time (user CPU time according to the UNIX time command) on a Sun Ultra-10 with a $440 \mathrm{MHz}$ Ultra-SPARC IIi processor and $640 \mathrm{MB}$ RAM, on the $816 \times 696$ Australian 6' DEM (including 100\% zero-padding) was 1:58 (min:sec) for the DoG wavelet, 22:19 for the Fan $\left(\Delta \theta=180^{\circ}\right)$, 2:05 for the Halo, 2:17 for the 
Morlet, 1:57 for the Paul, 2:13 for the Perrier, and 1:42 for the Poisson.

\section{readbin.m}

This Matlab script reads the binary files output by cwt2d.f, and also the file wavelengths. dat. The binary file should be renamed to, or copied to, a file called in. bin, or else the code may be altered to reflect the desired filename. Owing to the different byte-ordering styles on different computers, there are two lines of code for the fopen (read) stage: the undesired line should be commented out (with a \%). Other than this, all the Matlab code runs on both big-endian and little-endian machines. For users without Matlab, the author recommends taking a look at the format of the binary output files (the write statements towards the end of the main program), and writing your own script to read and plot.

\section{sliceplot.m}

This Matlab script will plot the 3D arrays generated by cwt2d.f, as in Fig. 15. The user has complete freedom in setting the position of the slices. The opening comments in sliceplot.m describe its usage. The program calls coords.m only when a new data set is read in: this stops the coordinates being allocated every time sliceplot.m is run. 
coords.m

This script is called by sliceplot.m, and does not need to be directly executed by the user. It initialises the $\mathrm{x}, \mathrm{y}$ and $\mathrm{z}$ coordinates, and finds their limits. This information is passed to sliceplot.m.

\section{plot2logpow.m}

A Matlab script to plot the data in the files log_pow_four.xy (radially-averaged Fourier power spectrum) and log_pow_wave.xy (global scalogram) output by cwt2d.f. The abscissa for both files is $\log _{10}$ (wavenumber, $\mathrm{rad} / \mathrm{m}$ ), while the ordinates are $\log _{10}$ (power). 